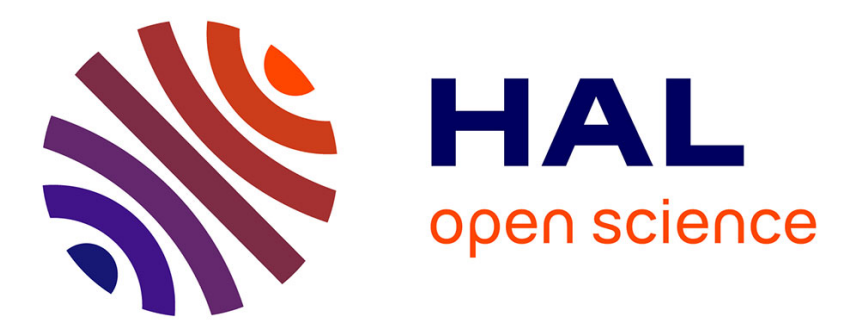

\title{
Elastic surface waves in crystals - part 2: cross-check of two full-wave numerical modeling methods
}

\author{
Dimitri Komatitsch, José M. Carcione, Fabio Cavallini, Nathalie
}

Favretto-Cristini

\section{- To cite this version:}

Dimitri Komatitsch, José M. Carcione, Fabio Cavallini, Nathalie Favretto-Cristini. Elastic surface waves in crystals - part 2: cross-check of two full-wave numerical modeling methods. Ultrasonics, 2011, 51 (8), pp.878-889. 10.1016/j.ultras.2011.05.001 . hal-00667712

HAL Id: hal-00667712

https://hal.science/hal-00667712

Submitted on 8 Feb 2012

HAL is a multi-disciplinary open access archive for the deposit and dissemination of scientific research documents, whether they are published or not. The documents may come from teaching and research institutions in France or abroad, or from public or private research centers.
L'archive ouverte pluridisciplinaire HAL, est destinée au dépôt et à la diffusion de documents scientifiques de niveau recherche, publiés ou non, émanant des établissements d'enseignement et de recherche français ou étrangers, des laboratoires publics ou privés. 


\title{
Elastic surface waves in crystals. Part 2: Cross-check of two full-wave numerical modeling methods
}

\author{
Dimitri Komatitsch ${ }^{\mathrm{a}, 1}$, José M. Carcione ${ }^{\mathrm{b}}$, Fabio Cavallini ${ }^{\mathrm{b}}$, Nathalie Favretto-Cristinic,** \\ ${ }^{a}$ Géosciences Environnement Toulouse, (UMR 5563) UR 154 CNRS Université Paul Sabatier, Observatoire \\ Midi-Pyrénées, 14 avenue Édouard Belin, 31400 Toulouse, France. \\ ${ }^{b}$ Istituto Nazionale di Oceanografia e di Geofisica Sperimentale, Borgo Grotta Gigante 42c, 34010 Sgonico, \\ Trieste, Italy. \\ ${ }^{c}$ Laboratoire de Mécanique et d'Acoustique (UPR 7051), CNRS, 31 chemin Joseph Aiguier, 13402 Marseille \\ Cedex 20, France.
}

\begin{abstract}
We obtain the full-wave solution for the wave propagation at the surface of anisotropic media using two spectral numerical modeling algorithms. The simulations focus on media of cubic and hexagonal symmetries, for which the physics has been reviewed and clarified in a companion paper. Even in the case of homogeneous media, the solution requires the use of numerical methods because the analytical Green's function cannot be obtained in the whole space. The algorithms proposed here allow for a general material variability and the description of arbitrary crystal symmetry at each grid point of the numerical mesh. They are based on high-order spectral approximations of the wave field for computing the spatial derivatives. We test the algorithms by comparison to the analytical solution and obtain the wave field at different faces (stress-free surfaces) of apatite, zinc and copper. Finally, we perform simulations in heterogeneous media, where no analytical solution exists in general, showing that the modeling algorithms can handle large impedance variations at the interface.
\end{abstract}

Keywords: Surface waves, anisotropy, modeling

\section{Introduction}

The problem of surface acoustic wave (SAW) propagation in anisotropic media has been studied for many decades. Nevertheless, anisotropy induces great difficulties in analytically and explicitly studying wave propagation because the anisotropic behavior of the medium considerably modifies the existence and the structure of the SAW that propagates at the free surface of the medium (see a companion paper [1] for a detailed review). Few problems in elastodynamics have a closed-form analytical solution and some can be investigated with semi-analytical methods, but often one cannot be sure if these methods give reliable solutions. Being able to accurately simulate wave propagation numerically is therefore essential in a wide range of fields,

\footnotetext{
* Corresponding author

Email addresses: dimitri.komatitsch@get.obs-mip.fr (Dimitri Komatitsch), jcarcione@inogs.it (José

M. Carcione), fcavallini@inogs.it (Fabio Cavallini), favretto@lma.cnrs-mrs.fr (Nathalie Favretto-Cristini) URL: http://www.univ-pau.fr/ dkomati1 (Dimitri Komatitsch)

${ }^{1}$ Also at Institut Universitaire de France, 103 boulevard Saint-Michel, 75005 Paris, France. Formerly at Université de Pau et des Pays de l'Adour, CNRS and INRIA, Laboratoire de Modélisation et d'Imagerie en Géosciences (UMR 5212) and IPRA, Avenue de l'Université, 64013 Pau Cedex, France.
} 
including ultrasonics, earthquake seismology and seismic prospecting. The emergence of ultrasonic techniques for nondestructive evaluation has provided a strong impulse to the study of wave propagation and its numerical simulation [2-8]. Ultrasonic theory and numerical modeling is applied to the detection of flaws and micro-cracks, inhomogeneous stress field evaluation, and the characterization of effective mechanical properties of fibers and composites with imperfect interface bonding. These systems generally possess anisotropic properties, described, in their most general form, by 21 elastic coefficients and by the mass density of the material. Numerical simulations therefore become an attractive method to describe the propagation of SAWs generated by a point source at a free surface that can be different from a symmetry plane of a given anisotropic medium and for which no analytical solution can be derived.

In the following sections we use two full-wave numerical methods to solve the problem without any approximation regarding the type of symmetry nor the orientation of the free surface. The methods are highly accurate because they are based on spectral representations of the wave field. We present some examples in hexagonal and cubic media, validation benchmarks against the analytical solution in known cases, and snapshots of propagation in more complex heterogeneous media.

\section{Equation of motion}

In a heterogeneous elastic, anisotropic medium, the linear wave equation may be written as

$$
\begin{aligned}
& \rho \ddot{\mathbf{u}}=\boldsymbol{\nabla} \cdot \boldsymbol{\sigma}+\mathbf{f}, \\
& \boldsymbol{\sigma}=\mathbf{C}: \boldsymbol{\varepsilon}, \\
& \boldsymbol{\varepsilon}=\frac{1}{2}\left[\boldsymbol{\nabla u}+(\boldsymbol{\nabla u})^{\top}\right],
\end{aligned}
$$

where $\mathbf{u}$ denotes the displacement vector, $\boldsymbol{\sigma}$ the symmetric, second-order stress tensor, $\boldsymbol{\varepsilon}$ the symmetric, second-order strain tensor, $\mathbf{C}$ the fourth-order stiffness tensor, $\rho$ the density, and $\mathbf{f}$ an external source force. A dot over a symbol denotes time differentiation, a colon denotes the tensor product, and a superscript $T$ denotes the transpose.

In the case of a fully anisotropic medium, the 3-D stiffness tensor $\mathbf{C}$ has 21 independent components. In 2-D, the number of independent components reduces to 6 . Using the reduced Voigt notation [9], Hooke's law may be written in the form

$$
\left(\begin{array}{l}
\sigma_{x x} \\
\sigma_{y y} \\
\sigma_{z z} \\
\sigma_{y z} \\
\sigma_{x z} \\
\sigma_{x y}
\end{array}\right)=\left(\begin{array}{llllll}
c_{11} & c_{12} & c_{13} & c_{14} & c_{15} & c_{16} \\
c_{21} & c_{22} & c_{23} & c_{24} & c_{25} & c_{26} \\
c_{31} & c_{32} & c_{33} & c_{34} & c_{35} & c_{36} \\
c_{41} & c_{42} & c_{43} & c_{44} & c_{45} & c_{46} \\
c_{51} & c_{52} & c_{53} & c_{54} & c_{55} & c_{56} \\
c_{61} & c_{62} & c_{63} & c_{64} & c_{65} & c_{66}
\end{array}\right)\left(\begin{array}{c}
\varepsilon_{x x} \\
\varepsilon_{y y} \\
\varepsilon_{z z} \\
2 \varepsilon_{y z} \\
2 \varepsilon_{x z} \\
2 \varepsilon_{x y}
\end{array}\right)
$$

Using this reduced notation, the stiffness matrix remains symmetric, i.e., $c_{I J}=c_{J I}$. The isotropic case is obtained by letting $c_{11}=c_{22}=c_{33}=\lambda+2 \mu, c_{12}=c_{13}=c_{23}=\lambda$ and $c_{44}=c_{55}=c_{66}=\mu$, where $\lambda$ and $\mu$ are the two Lamé parameters; all other coefficients are then equal to zero.

In the case of a medium with free surfaces, e.g., the edges of a crystal, or the surface of the Earth, the boundary condition is zero traction at the surface: $\boldsymbol{\sigma} \cdot \hat{\mathbf{n}}=\mathbf{0}$, where $\hat{\mathbf{n}}$ is the unit outward normal vector. The reader is referred for instance to Crampin et al.[10], Thomsen[11], Helbig[9] or Carcione[12] for further details on wave propagation in anisotropic media. 


\section{Time-domain modeling methods}

We propose algorithms to simulate surface waves in a material with arbitrary symmetry. The computations are based on two different numerical techniques, namely, the Fourier-Chebyshev pseudospectral method (PSM) [12-14] and the spectral finite-element method (SEM) [15-20]. The first is based on global differential operators in which the field is expanded in terms of Fourier and Chebyshev polynomials, while the second is an extension of the finite-element method that uses Legendre polynomials as interpolating functions. Both methods have spectral accuracy up to approximately the Nyquist wavenumber of the mesh. One of the aims of this work is to provide reliable techniques to compute numerical solutions in, e.g., crystals, metals and minerals for which analytical solutions do not exist. The proposed algorithms can obtain solutions for general heterogeneous media because the space is discretized on a mesh whose grid points can have varying values of the elastic properties, i.e., the medium can be inhomogeneous.

\subsection{The pseudospectral method}

The implementation of the pseudospectral method to simulate wave propagation in 2D and 3D unbounded anisotropic media is given in Carcione et al. [21] and Carcione et al. [22], respectively. The method including a free surface was first introduced by Kosloff et al. [23] for the 2D isotropic-elastic case. For computing spatial derivatives, the scheme is based on the Fourier and Chebyshev differential operators in the horizontal and vertical directions, respectively. These operators have infinite accuracy (within machine precision) up to two points per wavelength (the Nyquist wavenumber) and $\pi$ points per wavelength, respectively.

This modeling technique has been extended to the 3D anisotropic-elastic case by Tessmer [13] and to the 3D isotropic-anelastic case by Carcione et al. [24]. The first algorithm is used here to model surface waves. For completeness and ease in programming, we explicitly outline the equation of motion and the complete boundary treatment used in the calculations. The particle-velocity/stress formulation is

$$
\dot{\overline{\mathbf{v}}}=\mathbf{H} \cdot \overline{\mathbf{v}}+\mathbf{f},
$$

where

$$
\overline{\mathbf{v}}=\left(\mathbf{v}^{\top} ; \boldsymbol{\sigma}^{\top}\right)^{\top} \equiv\left(v_{x}, v_{y}, v_{z} ; \sigma_{x x}, \sigma_{y y}, \sigma_{z z}, \sigma_{y z}, \sigma_{x z}, \sigma_{x y}\right)^{\top}
$$

and

$$
\rho \mathbf{f}=\left(f_{x}, f_{y}, f_{z}, 0,0,0,0,0,0\right)^{\top}
$$

are the particle-velocity/stress and body-force vectors $(\mathbf{v}=\dot{\mathbf{u}})$,

$$
\mathbf{H}=\left(\begin{array}{cc}
\mathbf{0}_{3} & \rho^{-1} \nabla \\
\mathbf{C} \cdot \nabla^{\top} & \mathbf{0}_{6}
\end{array}\right),
$$

with

$$
\nabla=\left(\begin{array}{cccccc}
\partial_{x} & 0 & 0 & 0 & \partial_{z} & \partial_{y} \\
0 & \partial_{y} & 0 & \partial_{z} & 0 & \partial_{x} \\
0 & 0 & \partial_{z} & \partial_{y} & \partial_{x} & 0
\end{array}\right)
$$

and $\mathbf{O}_{n}$ denotes the zero matrix of dimension $n \times n$. Moreover, $\rho$ is the mass density. A numerical solution of equation (3) is obtained by means of a fourth-order Runge-Kutta method [12].

A less straightforward issue using pseudospectral differential operators is to model the freesurface boundary condition. While in finite-element methods the implementation of traction-free boundary conditions is natural - simply do not impose any constraint at the surface nodes - finitedifference and pseudospectral methods require a particular boundary treatment $[14,23,25,26]$. 
Free-surface and solid-solid boundary conditions can be implemented in numerical modeling with the Chebyshev method by using a boundary treatment based on characteristics variables [12]. Most explicit time integration schemes compute the operation $\mathbf{H} \cdot \overline{\mathbf{v}} \equiv(\overline{\mathbf{v}})^{\text {old }}$. The array $(\overline{\mathbf{v}})^{\text {old }}$ is then updated to give a new array $(\overline{\mathbf{v}})^{\text {new }}$ that takes the boundary conditions into account. Let us consider the boundary $z=0$ (e.g., the surface) and let us assume that the wave is incident on this boundary from the half-space $z>0$. The free surface conditions are obtained by computing the stresses from

$$
\sigma_{x x}^{(\text {new })}=\frac{b_{1}}{a_{14}} \quad \sigma_{y y}^{(\text {new })}=\frac{b_{2}}{a_{25}} \quad \sigma_{x y}^{(\text {new })}=\frac{b_{3}}{a_{37}}
$$

, where

$$
\left(\begin{array}{c}
b_{1} \\
b_{2} \\
b_{3}
\end{array}\right)=\left(\begin{array}{cccccc}
1 & 0 & a_{16} & 0 & a_{18} & a_{19} \\
0 & 1 & a_{26} & 0 & a_{28} & a_{29} \\
0 & 0 & a_{36} & 1 & a_{38} & a_{39}
\end{array}\right)\left(\begin{array}{c}
\sigma_{x x} \\
\sigma_{y y} \\
\sigma_{z z} \\
\sigma_{x y} \\
\sigma_{x z} \\
\sigma_{y z}
\end{array}\right)^{(\mathrm{old})}
$$

while the velocities are given by

$$
\left(\begin{array}{c}
v_{x} \\
v_{y} \\
v_{z}
\end{array}\right)^{(\text {new })}=\left(\begin{array}{c}
v_{x} \\
v_{y} \\
v_{z}
\end{array}\right)^{\text {(old) }}+\mathbf{A}^{-1} \mathbf{B}\left(\begin{array}{c}
\sigma_{z z} \\
\sigma_{x z} \\
\sigma_{y z}
\end{array}\right)^{(\text {old })}
$$

where

$$
\mathbf{A}=\left(\begin{array}{ccc}
a_{41} & a_{42} & a_{43} \\
a_{61} & a_{62} & a_{63} \\
a_{81} & a_{82} & a_{83}
\end{array}\right) \quad \text { and } \quad \mathbf{B}=\left(\begin{array}{ccc}
a_{46} & a_{48} & a_{49} \\
a_{66} & a_{68} & a_{69} \\
a_{86} & a_{88} & a_{89}
\end{array}\right)
$$

In the equations above, coefficients $a_{i j}$ depend only on the elastic coefficients and on density. Indeed, these coefficients may be computed based on the following three-step algorithm:

Step 1:. Define the matrix

$$
\mathbf{C}=\left(\begin{array}{ccc|cccccc}
0 & 0 & 0 & 0 & 0 & 0 & 0 & \rho^{-1} & 0 \\
0 & 0 & 0 & 0 & 0 & 0 & 0 & 0 & \rho^{-1} \\
0 & 0 & 0 & 0 & 0 & \rho^{-1} & 0 & 0 & 0 \\
\hline c_{15} & c_{14} & c_{13} & 0 & 0 & 0 & 0 & 0 & 0 \\
c_{25} & c_{24} & c_{23} & 0 & 0 & 0 & 0 & 0 & 0 \\
c_{35} & c_{34} & c_{33} & 0 & 0 & 0 & 0 & 0 & 0 \\
c_{56} & c_{46} & c_{36} & 0 & 0 & 0 & 0 & 0 & 0 \\
c_{55} & c_{45} & c_{35} & 0 & 0 & 0 & 0 & 0 & 0 \\
c_{45} & c_{44} & c_{34} & 0 & 0 & 0 & 0 & 0 & 0
\end{array}\right) .
$$


Step 2:: Compute the matrix $Q$ whose columns are the eigenvectors of matrix $\mathbf{C}$, and note that it has the form

$$
\mathbf{Q}=\left(\begin{array}{ccc|cc|cc|cc}
0 & 0 & 0 & p_{1} & -p_{1} & q_{1} & -q_{1} & r_{1} & -r_{1} \\
0 & 0 & 0 & p_{2} & -p_{2} & q_{2} & -q_{2} & r_{2} & -r_{2} \\
0 & 0 & 0 & p_{3} & -p_{3} & q_{3} & -q_{3} & r_{3} & -r_{3} \\
\hline 1 & 0 & 0 & p_{4} & p_{4} & q_{4} & q_{4} & r_{4} & r_{4} \\
0 & 1 & 0 & p_{5} & p_{5} & q_{5} & q_{5} & r_{5} & r_{5} \\
0 & 0 & 0 & p_{6} & p_{6} & q_{6} & q_{6} & r_{6} & r_{6} \\
0 & 0 & 1 & p_{7} & p_{7} & q_{7} & q_{7} & r_{7} & r_{7} \\
0 & 0 & 0 & p_{8} & p_{8} & q_{8} & q_{8} & r_{8} & r_{8} \\
0 & 0 & 0 & p_{9} & p_{9} & q_{9} & q_{9} & r_{9} & r_{9}
\end{array}\right)
$$

Step 3:. Compute the inverse of matrix $Q$, and note that it has the form

$$
\mathbf{Q}^{-\mathbf{1}}=\left(\begin{array}{rrr|rrrrrr}
0 & 0 & 0 & 1 & 0 & a_{16} & 0 & a_{18} & a_{19} \\
0 & 0 & 0 & 0 & 1 & a_{26} & 0 & a_{28} & a_{29} \\
0 & 0 & 0 & 0 & 0 & a_{36} & 1 & a_{38} & a_{39} \\
\hline a_{41} & a_{42} & a_{43} & 0 & 0 & a_{46} & 0 & a_{48} & a_{49} \\
-a_{41} & -a_{42} & -a_{43} & 0 & 0 & a_{46} & 0 & a_{48} & a_{49} \\
\hline a_{61} & a_{62} & a_{63} & 0 & 0 & a_{66} & 0 & a_{68} & a_{69} \\
-a_{61} & -a_{62} & -a_{63} & 0 & 0 & a_{66} & 0 & a_{68} & a_{69} \\
\hline a_{81} & a_{82} & a_{83} & 0 & 0 & a_{86} & 0 & a_{88} & a_{89} \\
-a_{81} & -a_{82} & -a_{83} & 0 & 0 & a_{86} & 0 & a_{88} & a_{89}
\end{array}\right) .
$$

The relationship between matrices (10) and (11) is given by

$$
\begin{aligned}
a_{16} & =\left[p_{9}\left(q_{8} r_{4}-q_{4} r_{8}\right)+p_{8}\left(q_{4} r_{9}-q_{9} r_{4}\right)+p_{4}\left(q_{9} r_{8}-q_{8} r_{9}\right)\right] / d_{1}, \\
a_{18} & =\left[p_{9}\left(q_{4} r_{6}-q_{6} r_{4}\right)+p_{6}\left(q_{9} r_{4}-q_{4} r_{9}\right)+p_{4}\left(q_{6} r_{9}-q_{9} r_{6}\right)\right] / d_{1}, \\
a_{19} & =\left[p_{8}\left(q_{6} r_{4}-q_{4} r_{6}\right)+p_{6}\left(q_{4} r_{8}-q_{8} r_{4}\right)+p_{4}\left(q_{8} r_{6}-q_{6} r_{8}\right)\right] / d_{1}, \\
a_{26} & =\left[p_{9}\left(q_{8} r_{5}-q_{5} r_{8}\right)+p_{8}\left(q_{5} r_{9}-q_{9} r_{5}\right)+p_{5}\left(q_{9} r_{8}-q_{8} r_{9}\right)\right] / d_{1}, \\
a_{28} & =\left[p_{9}\left(q_{5} r_{6}-q_{6} r_{5}\right)+p_{6}\left(q_{9} r_{5}-q_{5} r_{9}\right)+p_{5}\left(q_{6} r_{9}-q_{9} r_{6}\right)\right] / d_{1}, \\
a_{29} & =\left[p_{8}\left(q_{6} r_{5}-q_{5} r_{6}\right)+p_{6}\left(q_{5} r_{8}-q_{8} r_{5}\right)+p_{5}\left(q_{8} r_{6}-q_{6} r_{8}\right)\right] / d_{1}, \\
a_{36} & =\left[p_{9}\left(q_{8} r_{7}-q_{7} r_{8}\right)+p_{8}\left(q_{7} r_{9}-q_{9} r_{7}\right)+p_{7}\left(q_{9} r_{8}-q_{8} r_{9}\right)\right] / d_{1}, \\
a_{38} & =\left[p_{9}\left(q_{7} r_{6}-q_{6} r_{7}\right)+p_{7}\left(q_{6} r_{9}-q_{9} r_{6}\right)+p_{6}\left(q_{9} r_{7}-q_{7} r_{9}\right)\right] / d_{1}, \\
a_{39} & =\left[p_{8}\left(q_{6} r_{7}-q_{7} r_{6}\right)+p_{7}\left(q_{8} r_{6}-q_{6} r_{8}\right)+p_{6}\left(q_{7} r_{8}-q_{8} r_{7}\right)\right] / d_{1},
\end{aligned}
$$

together with

$$
\begin{array}{l|l|l}
a_{41}=\left(q_{2} r_{3}-q_{3} r_{2}\right) / d_{2} & a_{42}=\left(q_{3} r_{1}-q_{1} r_{3}\right) / d_{2} & a_{43}=\left(q_{1} r_{2}-q_{2} r_{1}\right) / d_{2}, \\
a_{46}=\left(q_{8} r_{9}-q_{9} r_{8}\right) / 2 d_{1} & a_{48}=\left(q_{9} r_{6}-q_{6} r_{9}\right) / 2 d_{1} & a_{49}=\left(q_{6} r_{8}-q_{8} r_{6}\right) / 2 d_{1}, \\
a_{61}=\left(p_{3} r_{2}-p_{2} r_{3}\right) / d_{2} & a_{62}=\left(p_{1} r_{3}-p_{3} r_{1}\right) / d_{2} & a_{63}=\left(p_{2} r_{1}-p_{1} r_{2}\right) / d_{2}, \\
a_{66}=\left(p_{9} r_{8}-p_{8} r_{9}\right) / 2 d_{1} & a_{68}=\left(p_{6} r_{9}-p_{9} r_{6}\right) / 2 d_{1} & a_{69}=\left(p_{8} r_{6}-p_{6} r_{8}\right) / 2 d_{1}, \\
a_{81}=\left(p_{2} q_{3}-p_{3} q_{2}\right) / d_{2} & a_{82}=\left(p_{3} q_{1}-p_{1} q_{3}\right) / d_{2} & a_{83}=\left(p_{1} q_{2}-p_{2} q_{1}\right) / d_{2}, \\
a_{86}=\left(p_{8} q_{9}-p_{9} q_{8}\right) / 2 d_{1} & a_{88}=\left(p_{9} q_{6}-p_{6} q_{9}\right) / 2 d_{1} & a_{89}=\left(p_{6} q_{8}-p_{8} q_{6}\right) / 2 d_{1},
\end{array}
$$

where

$$
\begin{aligned}
& d_{1}=p_{9}\left(q_{6} r_{8}-q_{8} r_{6}\right)+p_{8}\left(q_{9} r_{6}-q_{6} r_{9}\right)+p_{6}\left(q_{8} r_{9}-q_{9} r_{8}\right), \\
& d_{2}=2\left[p_{3}\left(q_{1} r_{2}-q_{2} r_{1}\right)+p_{2}\left(q_{3} r_{1}-q_{1} r_{3}\right)+p_{1}\left(q_{2} r_{3}-q_{3} r_{2}\right)\right]
\end{aligned}
$$


Finally, the non-reflecting (i.e., absorbing) boundary conditions arise from the following system of equations for the particle velocities

$$
\left(\begin{array}{lll}
a_{41} & a_{42} & a_{43} \\
a_{61} & a_{62} & a_{63} \\
a_{81} & a_{82} & a_{83}
\end{array}\right)\left(\begin{array}{c}
v_{x} \\
v_{y} \\
v_{z}
\end{array}\right)^{\text {(new) }}=-\frac{1}{2}\left(\begin{array}{c}
b_{5} \\
b_{7} \\
b_{9}
\end{array}\right),
$$

together with the following system of equations for the stresses

$$
\left(\begin{array}{llllll}
a_{14} & 0 & a_{16} & 0 & a_{18} & a_{19} \\
0 & a_{25} & a_{26} & 0 & a_{28} & a_{29} \\
0 & 0 & a_{36} & a_{37} & a_{38} & a_{39} \\
0 & 0 & a_{46} & 0 & a_{48} & a_{49} \\
0 & 0 & a_{66} & 0 & a_{68} & a_{69} \\
0 & 0 & a_{86} & 0 & a_{88} & a_{89}
\end{array}\right)\left(\begin{array}{c}
\sigma_{x x} \\
\sigma_{y y} \\
\sigma_{z z} \\
\sigma_{x y} \\
\sigma_{x z} \\
\sigma_{y z}
\end{array}\right)^{(\text {new })}=\left(\begin{array}{c}
b_{1} \\
b_{2} \\
b_{3} \\
b_{5} / 2 \\
b_{7} / 2 \\
b_{9} / 2
\end{array}\right)
$$

where

$$
\left(\begin{array}{c}
b_{5} \\
b_{7} \\
b_{9}
\end{array}\right)=-\mathbf{A}\left(\begin{array}{c}
v_{x} \\
v_{y} \\
v_{z}
\end{array}\right)^{(\text {old })}+\mathbf{B}\left(\begin{array}{c}
\sigma_{z z} \\
\sigma_{x z} \\
\sigma_{y z}
\end{array}\right)^{(\text {old })}
$$

with $\mathbf{A}$ and $\mathbf{B}$ given in (9), while $b_{1}, b_{2}$ and $b_{3}$ are given by (8).

\subsection{The spectral-element method}

In the spectral-element method (SEM), which is a continuous Galerkin approach, the strong form of the equations of motion (1) is first rewritten in a variational or weak formulation. Using such a variational approach has the direct advantage that the free-surface boundary condition at the surface of the model, which says that traction should be zero along the surface, is the natural boundary condition of the technique. Thus, one does not need to implement it explicitly, it is automatically enforced accurately. Because of that, the propagation of surface waves and their interaction with the shape of the surface of laboratory models can be computed in a very precise fashion [27]. This is true for geophysical models as well, for which the effect of complex topography on both surface waves and body waves can be accurately predicted [28].

The SEM being a full waveform modeling technique, it can compute terms that are often neglected in approximate methods, for instance the near-field terms [29]. Another advantage of that technique is that, contrary to finite-difference methods for instance, it does not need to resort to a staggered numerical grid in which different components of the strain tensor are defined at different locations; on the contrary, in the SEM all the components are defined at the same Gauss-Lobatto-Legendre grid point, and as a result the sum of all the strain terms required by Hooke's law in the case of general anisotropic media (2) can be performed without any additional interpolation. As a result, the SEM is very well suited to studying elastic wave propagation in complex anisotropic media [30-32].

Another important property of the SEM is the fact that it can be parallelized efficiently to take advantage of the distributed structure of modern supercomputers [33], and in particular on clusters of Graphics Processing Units (GPU) graphics cards [34-36], reaching speedup factors of more than an order of magnitude compared to a reference serial implementation on a CPU core; this makes it compare well in terms of performance to less flexible algorithms such as finite differences in the time domain (FDTD), which can also be implemented efficiently on GPUs $[37,38]$. 
Writing the variational form of the elastic wave equation is accomplished by dotting the strong (i.e., differential) form of the equation with an arbitrary test vector $\mathbf{w}$ and integrating by parts over the region of interest:

$$
\int_{\Omega} \rho \mathbf{w} \cdot \ddot{\mathbf{u}} \mathrm{d} \Omega+\int_{\Omega} \nabla \mathbf{w}: \mathbf{C}: \nabla \mathbf{u} \mathrm{d} \Omega=\int_{\Omega} \mathbf{w} \cdot \mathbf{f} \mathrm{d} \Omega+\int_{\Gamma_{\mathrm{abs}}} \mathbf{w} \cdot \mathbf{t} \mathrm{d} \Gamma,
$$

where $\mathbf{f}$ denotes the known external source force, $\mathbf{t}$ denotes the traction vector, and $\Omega$ denotes the domain under study, whose boundary $\Gamma$ usually consists in two parts: a boundary $\Gamma_{f}$ on which free-surface (i.e., traction-free) conditions are implemented, and an artificial boundary $\Gamma_{\text {abs }}$ used to truncate semi-infinite domains and on which outgoing waves must be absorbed. In the integration by part above, we have used the fact that the traction vanishes on the free boundaries $\Gamma_{f}$ of the domain and thus the related terms does not appear in the weak formulation because it is its natural boundary condition. In order to absorb outgoing waves on the fictitious edges of the mesh, Convolution Perfectly Matched absorbing Layers (C-PML) are implemented, see e.g. [39-41]; however in the case of elastic wave propagation in anisotropic crystals usually all the edges of the crystal are either free ('Neumann' boundary condition) or fixed/rigid with zero displacement ('Dirichlet' boundary condition) because the crystal is of finite size and thus no absorbing conditions need to be implemented.

To implement the Legendre spectral element discretization of the variational problem (12), one first needs to create a mesh of $n_{e l}$ non-overlapping hexahedra $\Omega_{e}$ on the domain $\Omega$, as in a classical finite element method (FEM). These elements are subsequently mapped to a reference cube $\Lambda=[-1,1]^{3}$ using an invertible local mapping $\mathcal{F}_{e}: \Lambda \rightarrow \Omega_{e}$, which enables one to go from the physical domain to the reference domain, and vice versa.

On the reference domain $\Lambda$, one introduces a set of local basis functions consisting of polynomials of degree $N$. On each element $\Omega_{e}$, mapped to the reference domain $\Lambda$, one then defines a set of nodes and chooses the polynomial approximation $\mathbf{u}_{N}^{e}$ and $\mathbf{w}_{N}^{e}$ of $\mathbf{u}$ and $\mathbf{w}$ to be the Lagrange interpolant at this set of nodes. These nodes $\xi_{i} \in[-1,1], i \in 0, \ldots, N$, are the GaussLobatto-Legendre (GLL) points which are the $(N+1)$ roots of

$$
\left(1-\xi^{2}\right) P_{N}^{\prime}(\xi)=0,
$$

where $P_{N}^{\prime}(\xi)$ is the derivative of the Legendre polynomial of degree $N$. On the reference domain $\Lambda$, the restriction of a given function $u_{N}$ to the element $\Omega_{e}$ can be expressed as

$$
u_{N}^{e}(\xi, \eta, \gamma)=\sum_{p=0}^{N} \sum_{q=0}^{N} \sum_{r=0}^{N} u_{N}^{e}\left(\xi_{p}, \eta_{q}, \gamma_{r}\right) h_{p}(\xi) h_{q}(\eta) h_{r}(\gamma)
$$

where $h_{p}(\xi)$ denotes the $p$-th 1-D Lagrange interpolant at the $(N+1)$ GLL points $\xi_{i}$ introduced above, which is by definition the unique polynomial of degree $N$ that is equal to one at $\xi=\xi_{p}$ and to zero at all other points $\xi=\xi_{q}$ for which $q \neq p$. From this definition, one obtains the crucial property

$$
h_{p}\left(\xi_{q}\right)=\delta_{p q},
$$

which will lead to a perfectly diagonal mass matrix.

After introducing the piecewise-polynomial approximation (14), the integrals in (12) can be approximated at the element level using the GLL integration rule:

$$
\begin{aligned}
\int_{\Omega} u_{N} w_{N} \mathrm{~d} \Omega & =\sum_{e=1}^{n_{e l}} \int_{\Omega_{e}} u_{N}^{e} w_{N}^{e} \mathrm{~d} \Omega \\
& \simeq \sum_{e=1}^{n_{e l}} \sum_{i=0}^{N} \omega_{i} \sum_{j=0}^{N} \omega_{j} \sum_{k=0}^{N} \omega_{k} J_{e}\left(\xi_{i}, \eta_{j}, \gamma_{k}\right) u_{N}^{e}\left(\xi_{i}, \eta_{j}, \gamma_{k}\right) w_{N}^{e}\left(\xi_{i}, \eta_{j}, \gamma_{k}\right) .
\end{aligned}
$$


The weights $\omega_{i}>0$ are independent of the element and are determined numerically [42], and $J_{e}$ is the Jacobian associated with the mapping $\mathcal{F}_{e}$ from the element $\Omega_{e}$ to the reference domain $\Lambda$.

Gradients are first computed in the reference domain $\Lambda$ :

$$
\begin{aligned}
\partial_{\xi} u_{N}^{e}(\xi, \eta, \gamma) & =\sum_{p=0}^{N} \sum_{q=0}^{N} \sum_{r=0}^{N} u_{N}^{e}\left(\xi_{p}, \eta_{q}, \gamma_{r}\right) h_{p}^{\prime}(\xi) h_{q}(\eta) h_{r}(\gamma), \\
\partial_{\eta} u_{N}^{e}(\xi, \eta, \gamma) & =\sum_{p=0}^{N} \sum_{q=0}^{N} \sum_{r=0}^{N} u_{N}^{e}\left(\xi_{p}, \eta_{q}, \gamma_{r}\right) h_{p}(\xi) h_{q}^{\prime}(\eta) h_{r}(\gamma), \\
\partial_{\gamma} u_{N}^{e}(\xi, \eta, \gamma) & =\sum_{p=0}^{N} \sum_{q=0}^{N} \sum_{r=0}^{N} u_{N}^{e}\left(\xi_{p}, \eta_{q}, \gamma_{r}\right) h_{p}(\xi) h_{q}(\eta) h_{r}{ }^{\prime}(\gamma),
\end{aligned}
$$

where $h^{\prime}$ denotes the derivative of the 1-D Lagrange interpolant. One subsequently uses the chain rule to compute the derivatives in the physical domain, i.e.,

$$
\begin{aligned}
\partial_{x} & =\xi_{x} \partial_{\xi}+\eta_{x} \partial_{\eta}+\gamma_{x} \partial_{\gamma}, \\
\partial_{y} & =\xi_{y} \partial_{\xi}+\eta_{y} \partial_{\eta}+\gamma_{y} \partial_{\gamma}, \\
\partial_{z} & =\xi_{z} \partial_{\xi}+\eta_{z} \partial_{\eta}+\gamma_{z} \partial_{\gamma},
\end{aligned}
$$

where the components of the Jacobian matrix, $\xi_{x}, \xi_{y}, \xi_{z}$ etc... are computed based upon the mapping $\mathcal{F}_{e}$.

The effects of anisotropy in (12) are included in the term $\int_{\Omega} \boldsymbol{\nabla} \mathbf{w}: \mathbf{C}: \boldsymbol{\nabla} \mathbf{u} \mathrm{d} \Omega$, which can be rewritten as $\int_{\Omega} \boldsymbol{\sigma}\left(\mathbf{u}_{N}\right): \nabla \mathbf{w}_{N} \mathrm{~d} \Omega$. Written out explicitly, the integrand is

$$
\boldsymbol{\sigma}\left(\mathbf{u}_{N}\right): \nabla \mathbf{w}_{N}=\sigma_{i j} \partial_{j} w_{i}
$$

In the fully anisotropic 3 -D case, using the definition $\varepsilon_{i j}=\left(\partial_{i} u_{j}+\partial_{j} u_{i}\right) / 2$, Hooke's law $(2)$, when injected in (19) to obtain the developed expression of $\boldsymbol{\sigma}\left(\mathbf{u}_{N}\right): \nabla \mathbf{w}_{N}$, gives a sum of terms of the form $c_{\alpha \beta} \partial_{a} u_{b} \partial_{c} w_{d}$, with $c_{\alpha \beta}$ the components of the reduced stiffness matrix in (2). Each of these terms, integrated over an element $\Omega_{e}$, is easily computed by substituting the expansion of the fields (14), computing gradients using (17) and the chain rule (18), and using the GLL integration rule (16).

After this spatial discretization with spectral elements, imposing that (12) holds for any test vector $\mathbf{w}_{N}$, as in a classical FEM, we have to solve an ordinary differential equation in time. Denoting by $\overline{\mathbf{u}}$ the global vector of unknown displacement in the medium, we can rewrite equation (12) in matrix form as

$$
\mathbf{M} \ddot{\overline{\mathbf{u}}}+\mathbf{K} \overline{\mathbf{u}}=\overline{\mathbf{f}},
$$

where $\mathbf{M}$ is called the mass matrix, $\mathbf{K}$ the stiffness matrix, and $\overline{\mathbf{f}}$ the source term. A very important property of the Legendre SEM used here from an implementation point of view, which allows for a drastic reduction in the complexity and the cost of the algorithm, is the fact that the mass matrix $M$ is diagonal; this stems from the choice of Lagrange interpolants at the GLL points in conjunction with the GLL integration rule, which results in (15). This constitutes a significant difference compared to a classical FEM and to the Chebyshev SEM of Patera[15] and of e.g. Priolo et al.[43]. As a result, fully explicit time evolution schemes can be used.

Time discretization of the second-order ordinary differential equation in time (20) is performed based on a classical explicit Newmark centered finite-difference scheme[44], which is second-order accurate and conditionally stable. We assume zero initial conditions for the displacement and velocity fields, i.e., the medium is initially at rest. Higher-order time schemes can be used if 


\begin{tabular}{|l|c|c|c|c|c|c|c|}
\hline Material & $\begin{array}{c}c_{11} \\
(\mathrm{GPa})\end{array}$ & $\begin{array}{c}c_{12} \\
(\mathrm{GPa})\end{array}$ & $\begin{array}{c}c_{13} \\
(\mathrm{GPa})\end{array}$ & $\begin{array}{c}c_{33} \\
(\mathrm{GPa})\end{array}$ & $\begin{array}{c}c_{55} \\
(\mathrm{GPa})\end{array}$ & $\begin{array}{c}\rho \\
\left(\mathrm{kg} / \mathrm{m}^{3}\right)\end{array}$ & Symmetry \\
\hline Apatite & 167 & 13.1 & 66 & 140 & 66.3 & 3190 & hexagonal \\
\hline Beryllium & 292 & 26.7 & 14 & 336 & 162 & 1848 & hexagonal \\
\hline Zinc & 165 & 31 & 50 & 62 & 39.6 & 7140 & hexagonal \\
\hline Copper & 169 & 122 & $c_{12}$ & $c_{11}$ & 75.3 & 8920 & cubic \\
\hline Epoxy & 7.17 & $c_{11}-2 c_{55}$ & $c_{12}$ & $c_{11}$ & 1.61 & 1120 & isotropic \\
\hline
\end{tabular}

Table 1: Elastic constants and density of the different materials used in this study.

needed, for instance fourth-order Runge-Kutta or symplectic schemes [45, 46]; this can be useful in particular for simulations comprising a very large number of time steps, for which the fact that the spatial SEM discretisation is of high order while the time discretisation is only second order implies that overall accuracy is significantly reduced because of the time scheme.

\section{Numerical simulations}

We consider the materials whose properties are given in Table 1, and which are dissimilar: apatite, beryllium and zinc have hexagonal symmetry and copper has cubic symmetry, with $c_{22}=c_{11}$, while epoxy is isotropic.

The pseudospectral method uses a mesh composed of 81 grid points along the three Cartesian directions, with a constant grid spacing of $2.5 \mathrm{~mm}$ along the $x$ - and $y$-directions and a total mesh size of $20 \mathrm{~cm}$ in the $z$-direction with varying grid spacing. The surface of the sample is the $(x, y, z=0)$-plane. The source is a vertical force located at the surface and has the time history

$$
h(t)=\cos \left[2 \pi\left(t-t_{0}\right) f_{0}\right] \exp \left[-2\left(t-t_{0}\right)^{2} f_{0}^{2}\right]
$$

where $f_{0}$ is the dominant frequency and $t_{0}=3 /\left(2 f_{0}\right)+5 \cdot 10^{-6} \mathrm{~s}$ is a onset delay time that we use in order to ensure zero initial conditions. The time step of the Runge-Kutta algorithm is $0.05 \mu \mathrm{s}$ for apatite and $0.1 \mu \mathrm{s}$ for zinc and copper. Figures 1,2 and 3 show the wavefronts (energy velocities) in an unbounded medium and snapshots in the $(x, z)$-plane for apatite, zinc and copper. The dominant frequencies of the source are $250 \mathrm{kHz}, 150 \mathrm{kHz}$ and $200 \mathrm{kHz}$, with total propagation times of $25 \mu \mathrm{s}, 50 \mu \mathrm{s}$ and $40 \mu \mathrm{s}$, respectively.

The Rayleigh wave can be observed at the surface, and the $\mathrm{qP}$ and $\mathrm{qS}$ waves in the interior of the medium. Figure 4 shows spectral-element snapshots at different propagation times at the surface of copper, i.e., in the (001)-plane in terms of Miller indices, where the Rayleigh wavefront can be seen [47].

The mesh contains a total of $90 \times 90 \times 90$ spectral elements and we use polynomial basis functions of degree $N=4$. The total size of the mesh is $40 \mathrm{~cm} \times 40 \mathrm{~cm} \times 40 \mathrm{~cm}$ and we use a time step of $0.03 \mu \mathrm{s}$. Apatite and zinc are azimuthally isotropic in this surface and therefore the wavefront is isotropic. Snapshots for the pseudospectral method of the wave field at the surface of apatite, with the sample rotated by an angle of $\pi / 4$ and then an angle of $\pi / 2$ with respect to the surface are displayed in Figure 5. The surfaces are the (101)- and (100)-planes in terms of Miller indices. In this case, the anisotropy of the Rayleigh wave can clearly be observed.

The analytical solution [48] for the three-dimensional Green's function (i.e., the impulse response) for a surface source and a receiver located along a vertical line below the source, in the interior of the medium, is represented in Figure 6.

For completeness the analytical expression to compute it is given in Appendix A. It corresponds to the Green function computed $15 \mathrm{~cm}$ below the surface for apatite and then for 

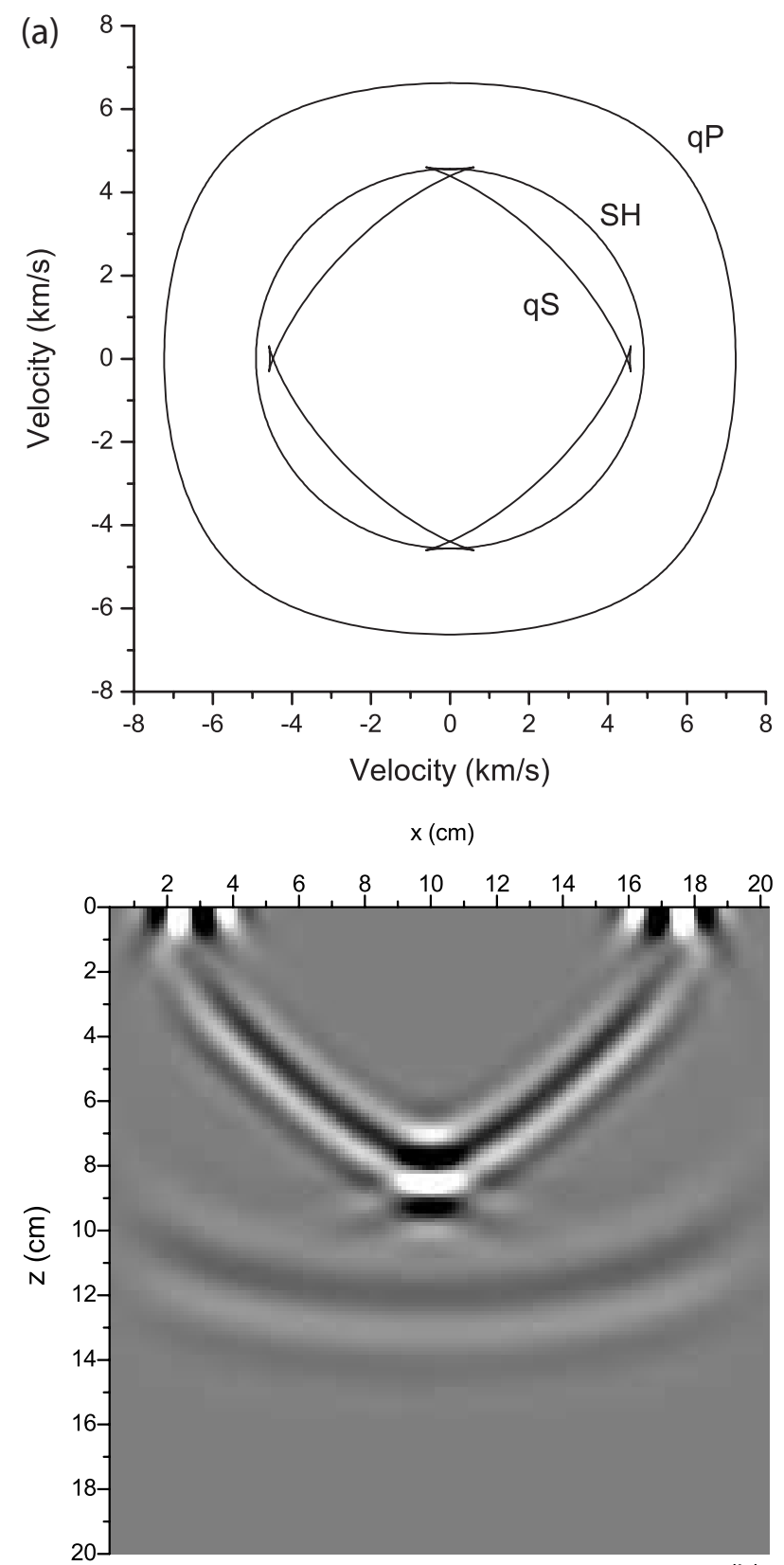

(b)

Figure 1: Wave fronts in apatite (a) and corresponding snapshot computed using the pseudospectral technique (b) at the $(x, z)$-plane containing the source, which is located at the surface. 

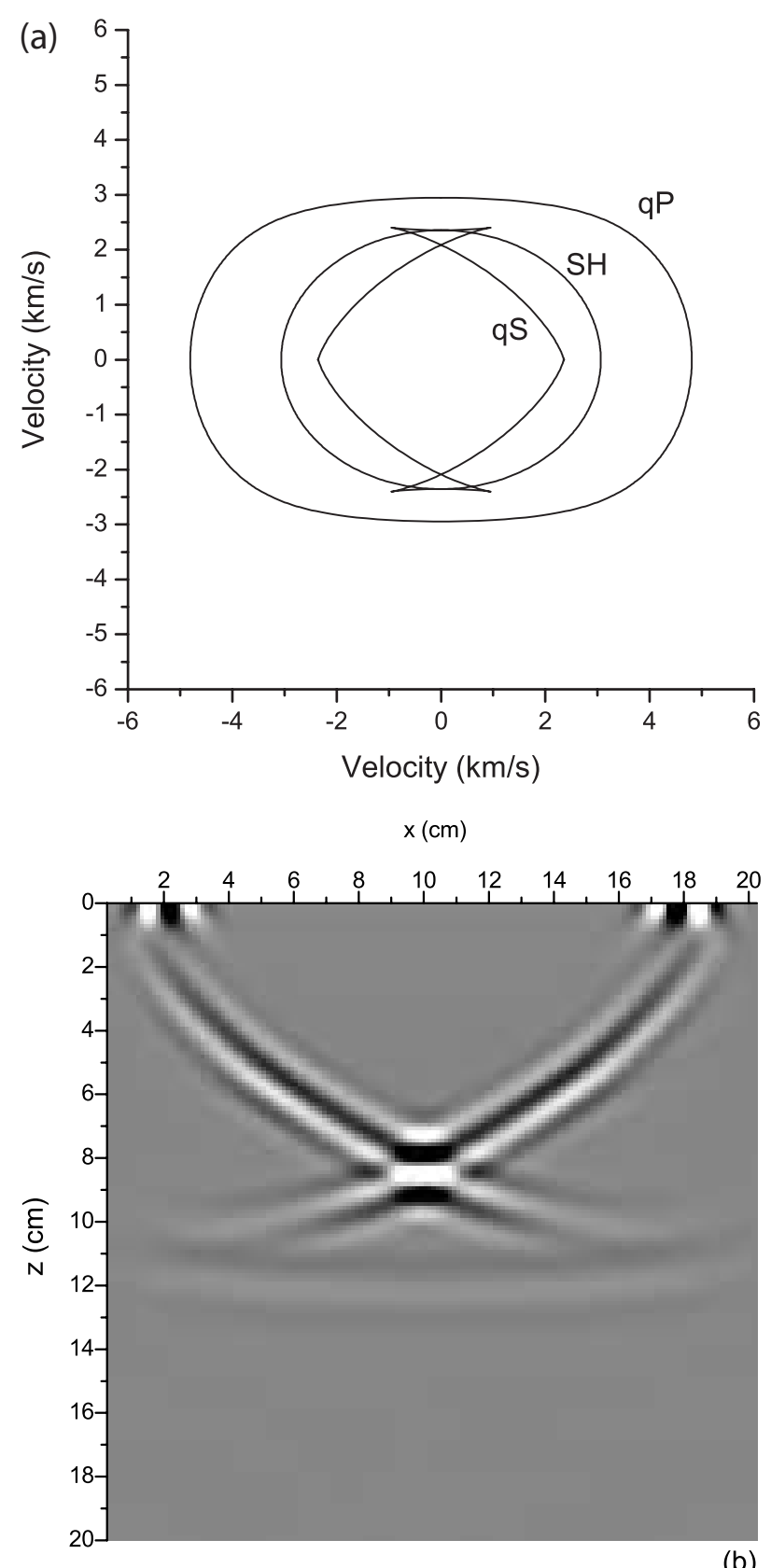

Figure 2: Same as Figure 1 for zinc. 

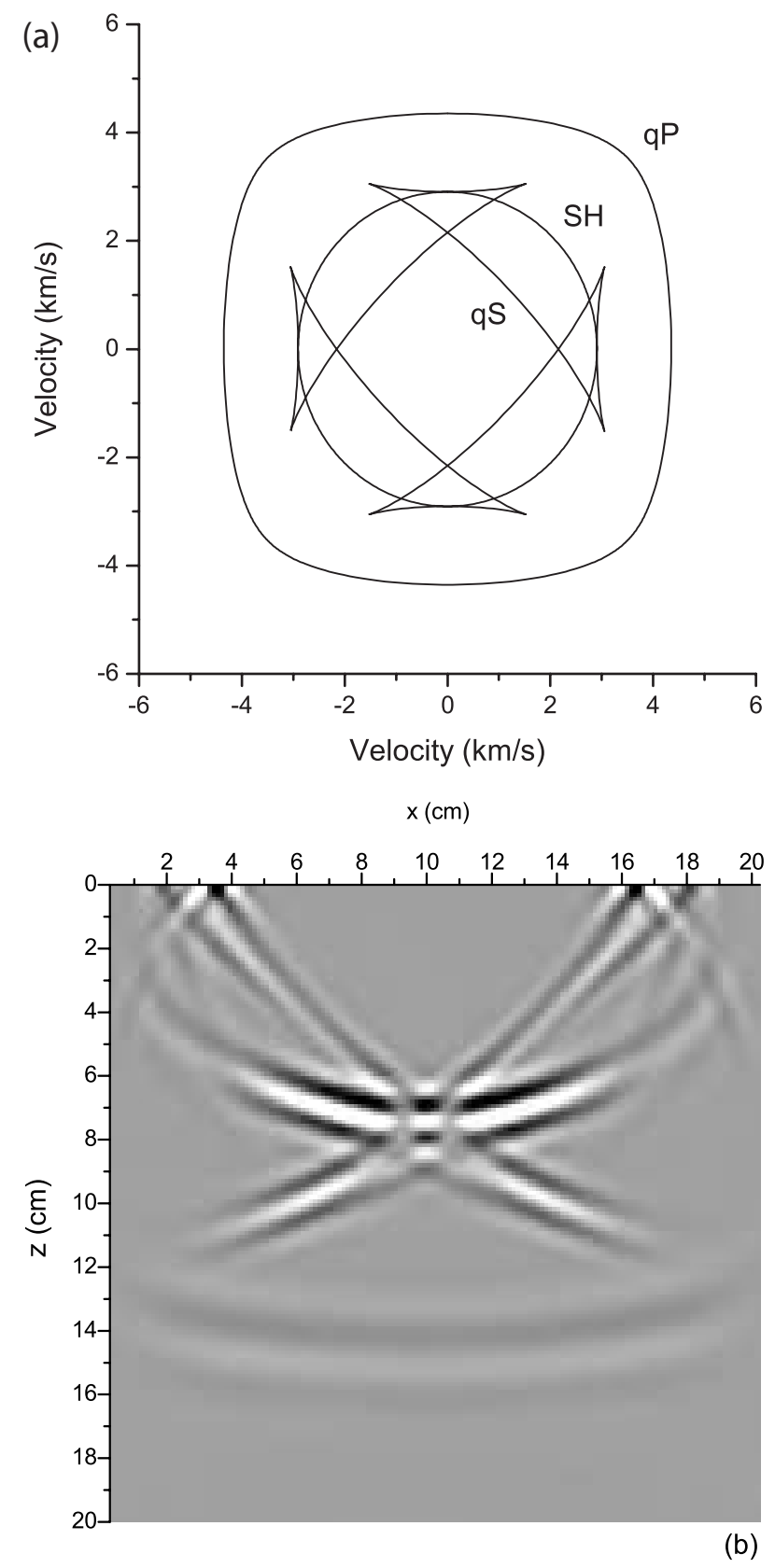

Figure 3: Same as Figure 1 for copper. 

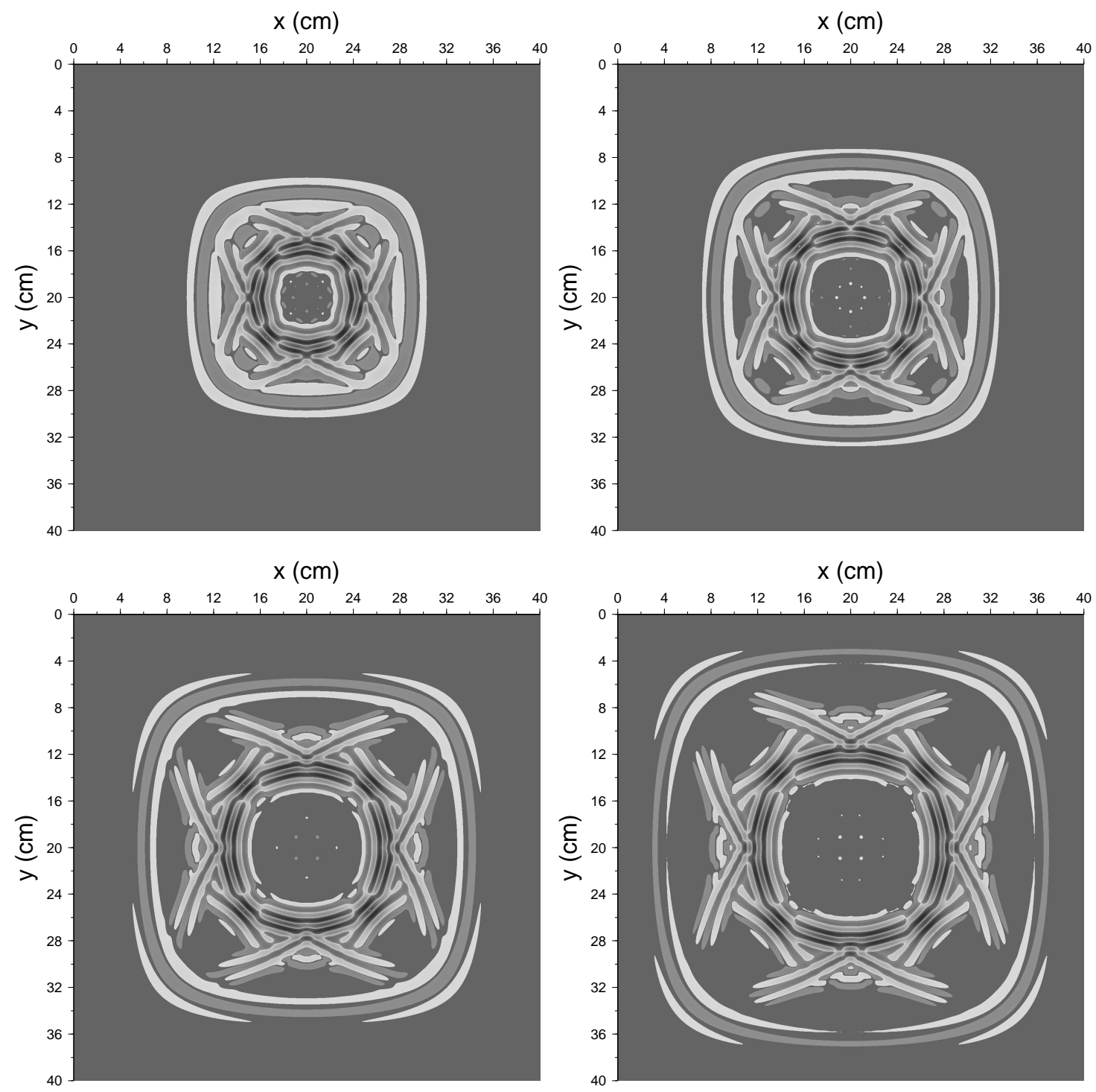

Figure 4: Snapshots at propagation times of $28 \mu \mathrm{s}, 34 \mu \mathrm{s}, 40 \mu \mathrm{s}$ and $46 \mu \mathrm{s}$ computed using the spectral-element technique at the surface of a three-dimensional copper crystal, i.e., in the $(x, y)$-plane. 

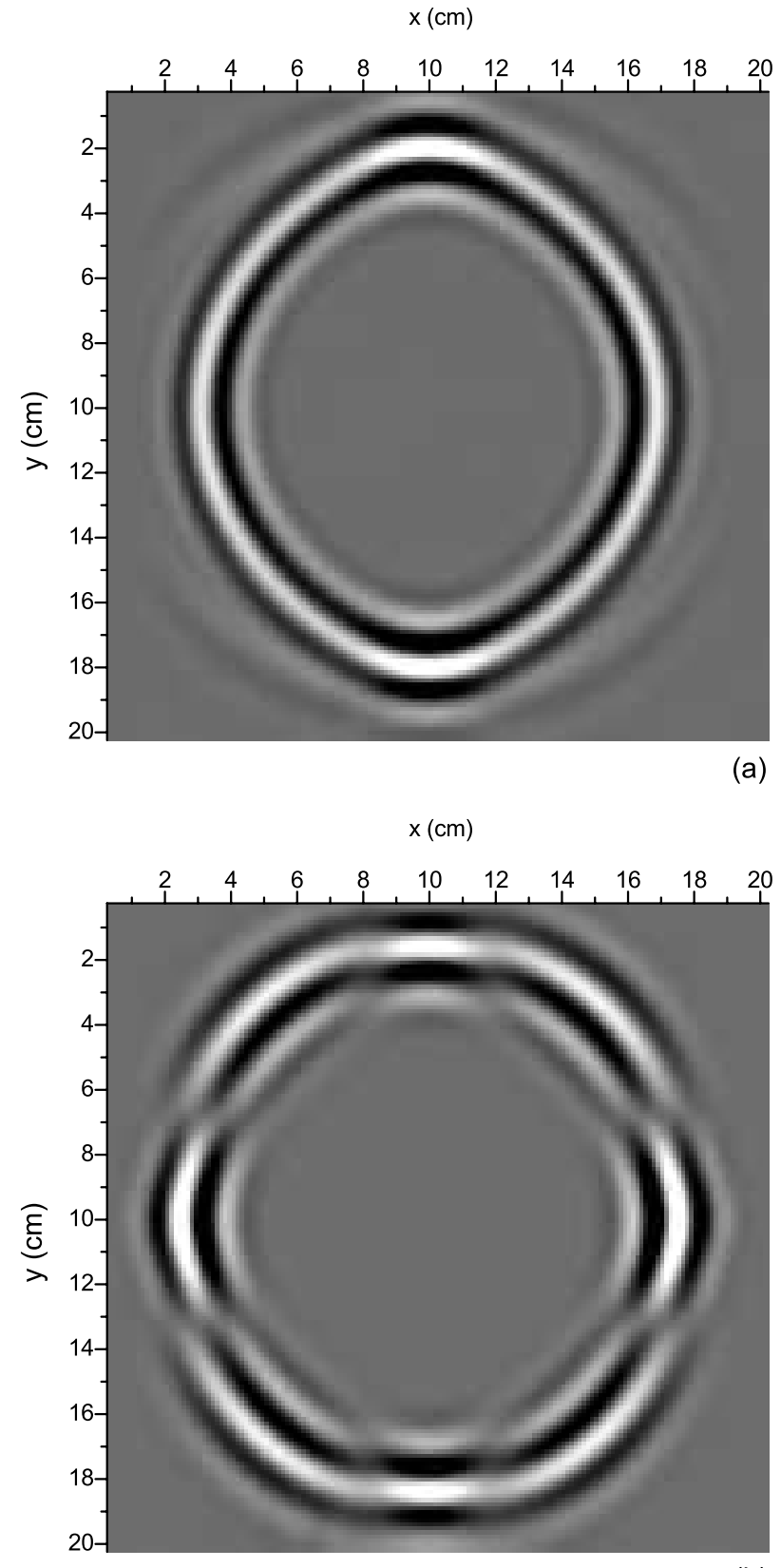

(b)

Figure 5: Snapshots computed using the pseudospectral technique at the surface of apatite, with the symmetry axis making an angle $\pi / 4$ (a) and an angle $\pi / 2$ (b) with the surface. 

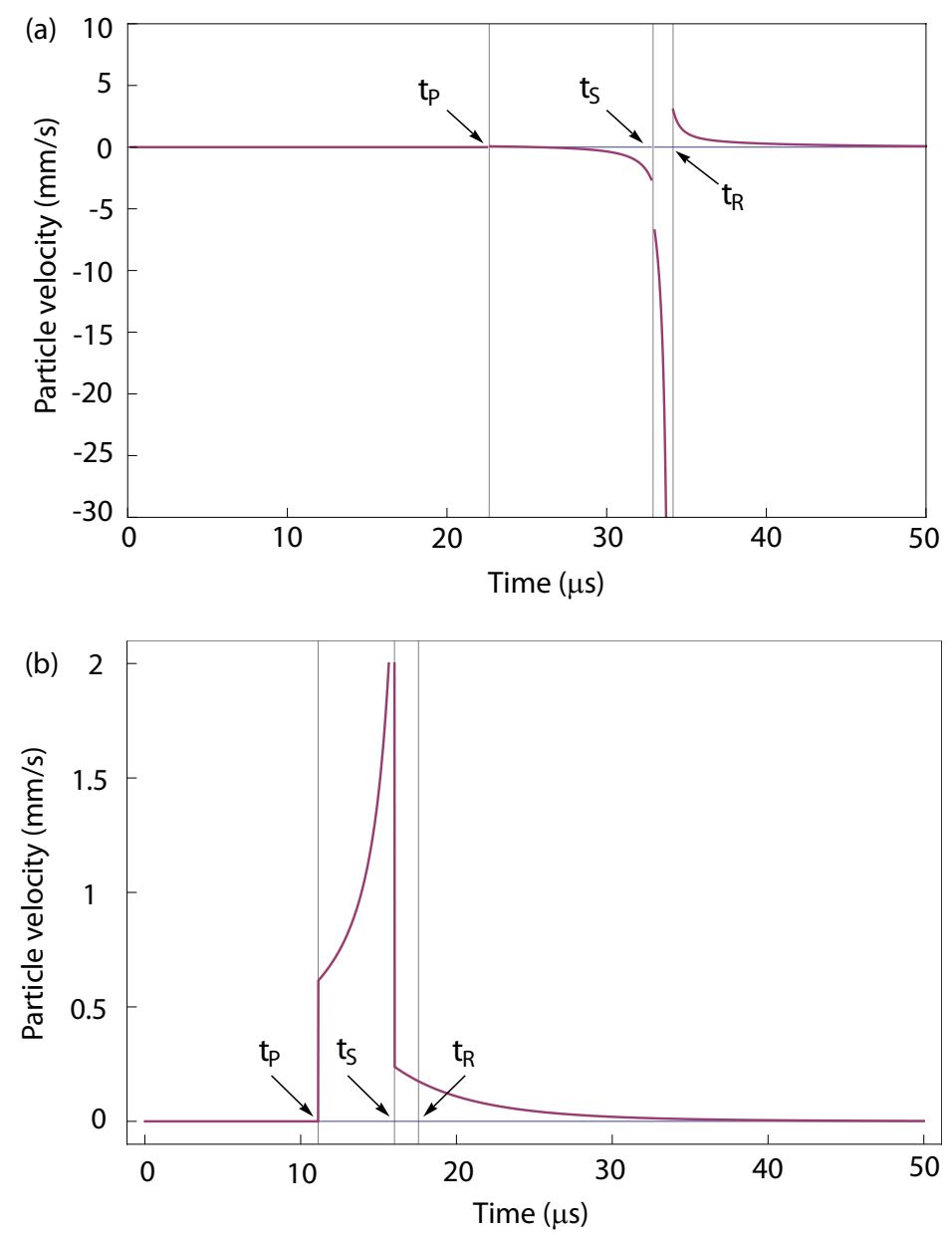

Figure 6: Analytical three-dimensional Green's function (i.e., impulse response) for apatite (a) and beryllium (b) computed $15 \mathrm{~cm}$ below the source. Symbols $t_{P}$ and $t_{R}$ denote the arrival time of qP and surface waves, respectively, while $t_{S}$ is the arrival time of $\mathrm{SH}$ and qSV modes. 

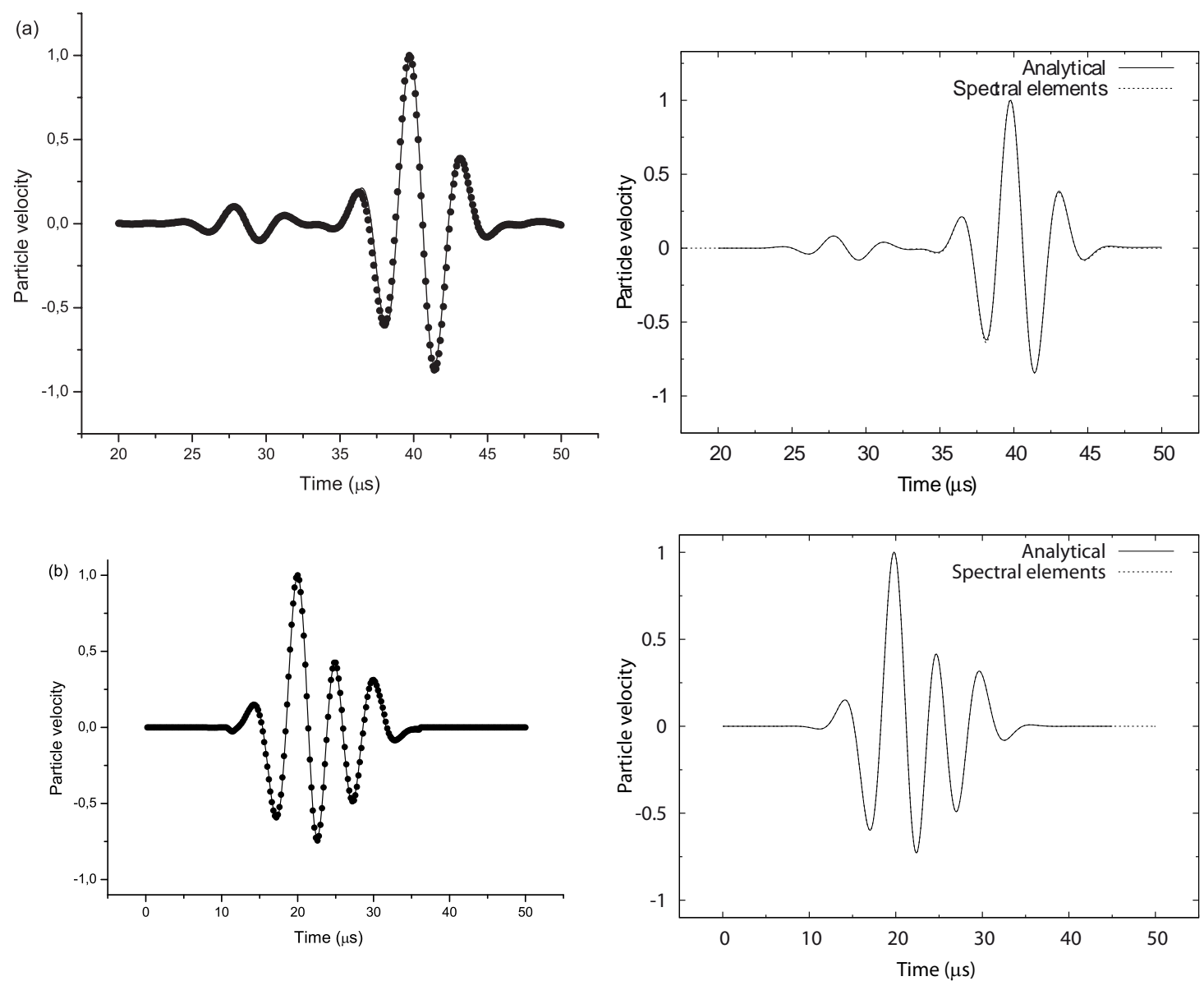

Figure 7: Left column: Comparison of analytical (solid line) and numerical (black dots) solutions for the vertical component of the velocity vector for apatite (a) and beryllium (b) in the case of the pseudspectral numerical technique. The source-receiver locations are those of Figure 6. The dominant frequency of the source is $250 \mathrm{kHz}$ for apatite in (a) and $150 \mathrm{kHz}$ for beryllium in (b). Right column: same comparison but for the analytical solution (solid line) versus the spectral-element numerical solution (dotted line).

beryllium. The P- and S-wave velocities of beryllium are almost twice that of other metals, i.e., $13484 \mathrm{~m} / \mathrm{s}$ and $9363 \mathrm{~m} / \mathrm{s}$ along the symmetry axis, respectively. These high velocities allow us to use a larger grid spacing of $4.4 \mathrm{~mm}$ in the $x$ - and $y$-directions for the pseudospectral technique and a total mesh size of $35 \mathrm{~cm}$ in the $z$-direction, keeping the same time step as that used for apatite, i.e., $0.05 \mu \mathrm{s}$; using a smaller grid allows us to save in terms of computational cost. Validation tests for both modeling algorithms versus the analytical solution convolved with the source time history (21) for apatite and beryllium are shown in Figure 7. The fit obtained is excellent for both techniques.

In the case of the spectral-element method, the mesh contains a total of $60 \times 60 \times 60$ spectral elements and we use polynomial basis functions of degree $N=4$. The total size of the mesh is $40 \mathrm{~cm} \times 40 \mathrm{~cm} \times 40 \mathrm{~cm}$ in the case of apatite and $60 \mathrm{~cm} \times 60 \mathrm{~cm} \times 60 \mathrm{~cm}$ in the case of beryllium, and we use a time step of $0.03 \mu \mathrm{s}$.

The practical applications of numerical modeling are numerous. One of them is to use it as a research tool to numerically investigate the complex behavior of waves propagating in crystals 


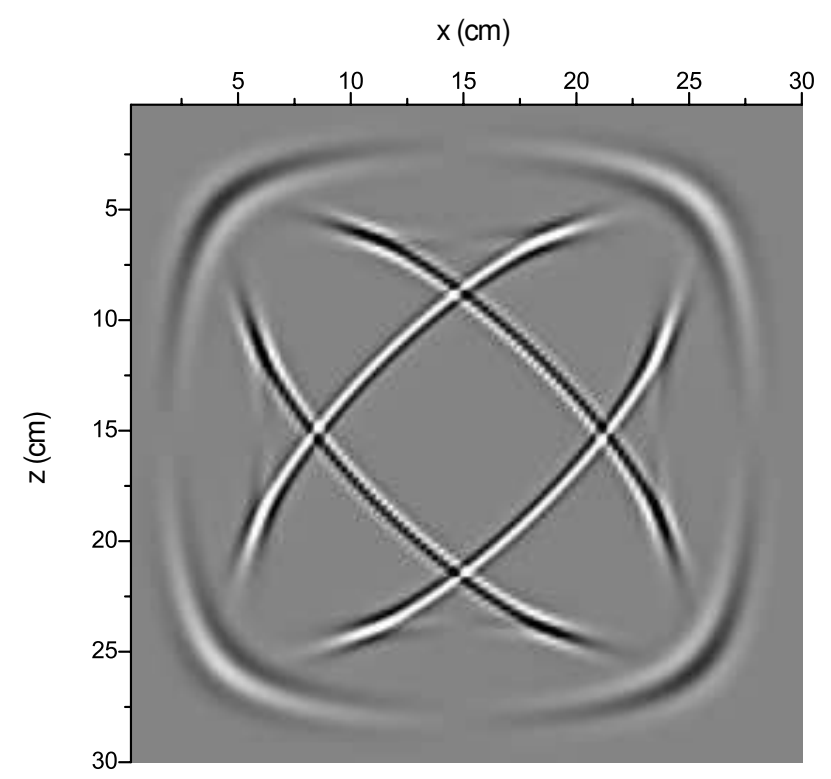

Figure 8: Snapshot of a two-dimensional simulation for a copper crystal computed using the staggered Fourier spectral technique of Carcione [52]. When comparing to Figure 3a, one can observe that the ends of the qS cusps extend as diffraction phenomena forming two quasi-ellipses.

when an analytical or closed form solution is not available. Recently, Deschamps and his collaborators [49-51] showed that the cuspidal triangles of the qS wave extend beyond the edges or vertices of the cuspidal triangles, and that this phenomenon can be explained by inhomogeneous plane waves. In order to show this, we perform a simulation using a two-dimensional qP-qS modeling algorithm based on the staggered Fourier method to compute the spatial derivatives [52]. The mesh has $120 \times 120$ grid points with a constant grid spacing of $2.5 \mathrm{~mm}$. A vertical force with a dominant frequency of $200 \mathrm{kHz}$ is applied at its center. Figure 8 shows a snapshot at a time of $36 \mu \mathrm{s}$ for a copper crystal; the $\mathrm{qP}$ and $\mathrm{qS}$ waves can be seen (outer and inner wavefronts, respectively). When comparing to Figure 3a, one can observe that the ends of the qS cusps extend as diffraction phenomena forming two quasi-ellipses.

Because the simulation is two dimensional and the source is placed in the center of the model, it contains no surface waves, only body waves. This explains why this snapshot looks different from the snapshots of Figure 4, which are dominated by surface waves. Indeed, as the simulation illustrated in Figure 4 is three dimensional with the source located exactly at the surface, Figure 4 thus not only has body waves as in Figure 3a, but also surface waves superimposed and dominant.

The proposed modeling algorithms can handle heterogeneous media, therefore numerical simulations can be performed in cases for which there is no known analytical solution. In the next simulation we consider a zinc sample coated with a substrate of epoxy of $5 \mathrm{~mm}$ thickness. Epoxy is isotropic and has the elastic constants given in Table 1. The simulation uses the same numerical parameters as those used to generate Figure 2, but the vertical force source is located at a depth of $1 \mathrm{~cm}$ in the zinc crystal. In the snapshots of Figure 9, isotropic and dispersive Rayleigh wavefronts can be seen at the surface, and we notice that most of the energy is contained in the thin substrate.

The model shown in Figure 10a is composed of a prism of epoxy embedded in zinc. The numerical parameters are unchanged and the source is a combination of three directional forces applied at the location indicated by a star in the model. Figures $10 \mathrm{~b}$ and $10 \mathrm{c}$ show snapshots at 


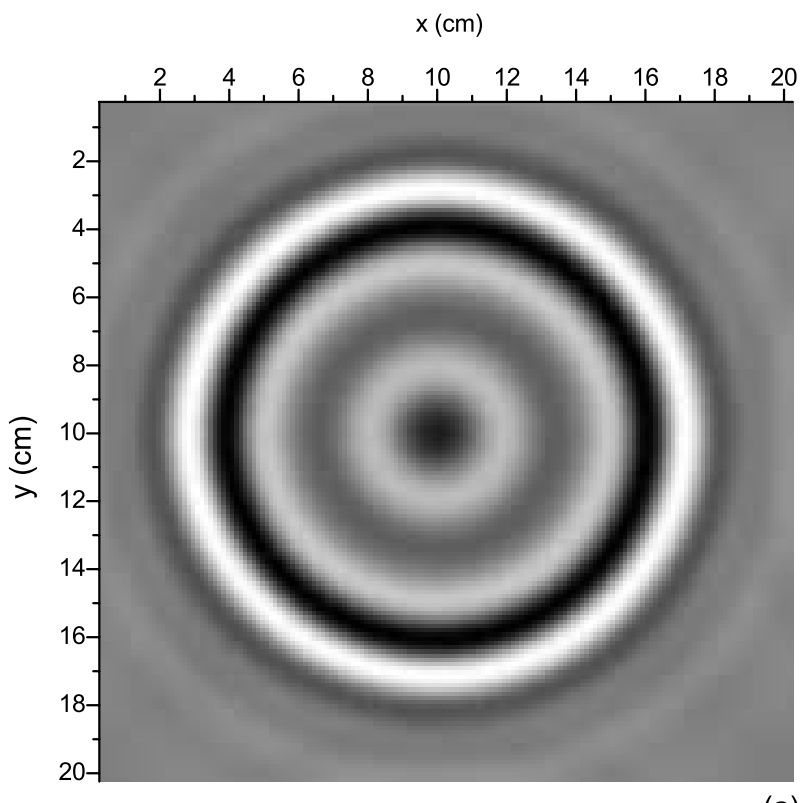

(a)

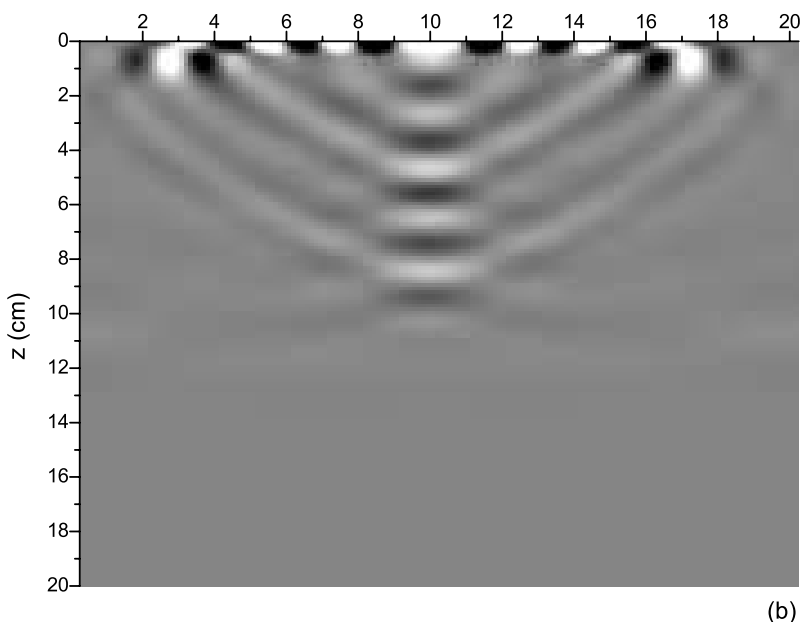

Figure 9: Snapshot computed using the pseudospectral technique at the surface (a) and at a vertical section (b). The medium is zinc overlaid by a substrate of epoxy of $5 \mathrm{~mm}$ thickness. 
the surface and at vertical section at a time of $55 \mu \mathrm{s}$, respectively. Most of the energy is trapped in the epoxy prism.

Figure 11 shows snapshots at a time of $50 \mu$ s at the same planes but replacing the epoxy prism with a copper prism. The impedance contrast between the two media is weaker and therefore energy trapping is much reduced.

\section{Conclusions}

The two numerical modeling methods compute the full wave field and have spectral accuracy. At each grid point these methods allow us to model an anisotropic medium of arbitrary crystal symmetry, i.,e., a triclinic medium or a medium of lower symmetry whose symmetry axes can be rotated by any angle. We have shown numerical examples for media of hexagonal or cubic symmetry, for which we obtained time histories and snapshots at the surface and at vertical sections. The wavefronts have been compared with the ray surfaces (energy or group velocities) obtained based on a plane-wave analysis. The modeling algorithms have been successfully tested against the analytical solution for a point force source located at the surface of a crystal and a receiver located in the interior of the medium. We have shown how these modeling tools can be used to simulate phenomena predicted by plane-wave analyses, as the continuation of the cuspidal triangles of the qS wave in cubic crystals. Moreover, we have simulated wave propagation in the presence of a free surface in cases where there is no analytical solution for models composed of media of dissimilar crystal symmetry and with contrasting elastic properties.

\section{Acknowledgements}

The authors thank Arthur G. Every for fruitful discussion.

Some of the calculations were performed on an SGI cluster at Centre Informatique National de l'Enseignement Suprieur (CINES) in Montpellier, France. This material is based in part upon research supported by European FP6 Marie Curie International Reintegration Grant MIRG-CT2005-017461. 

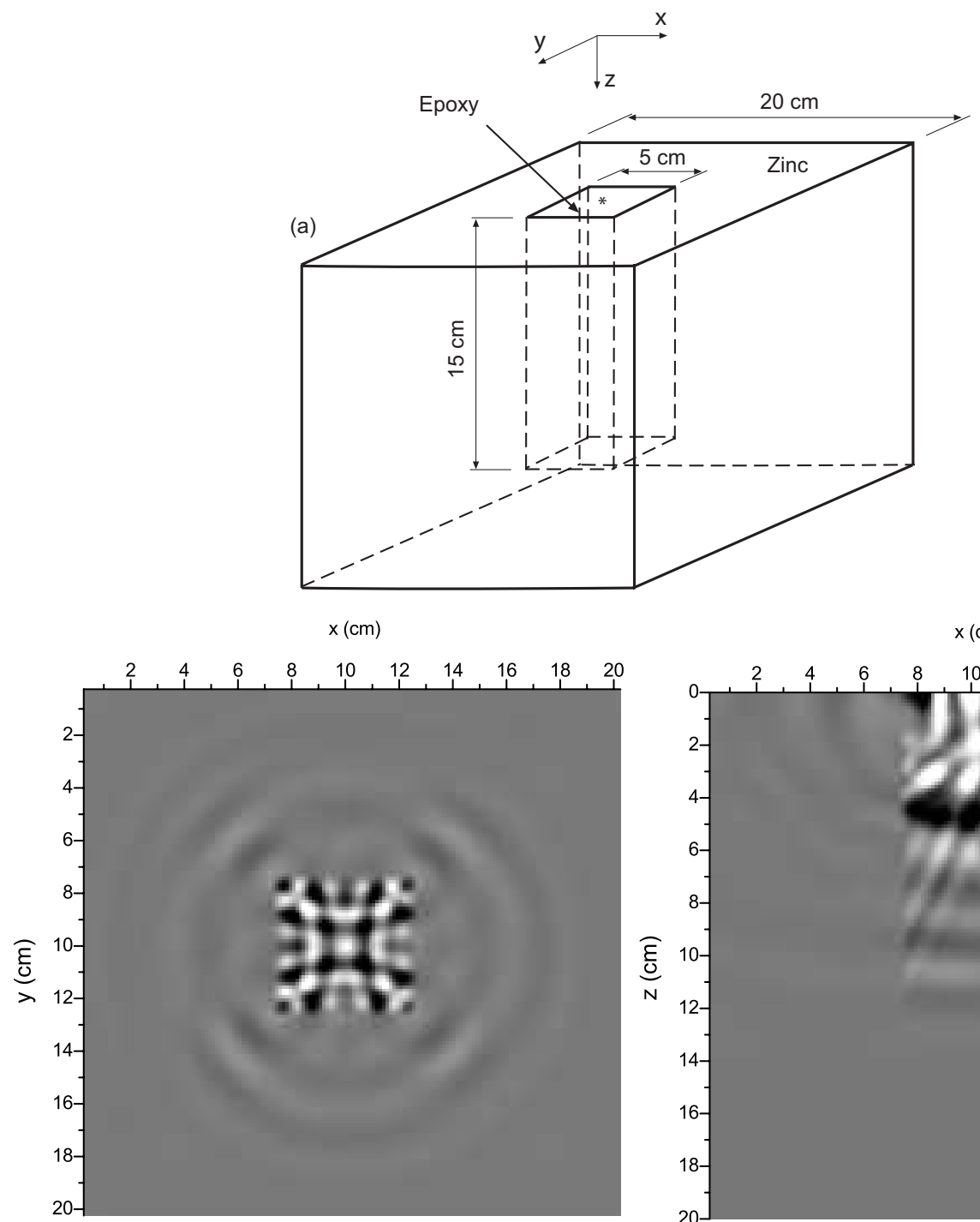

(b)

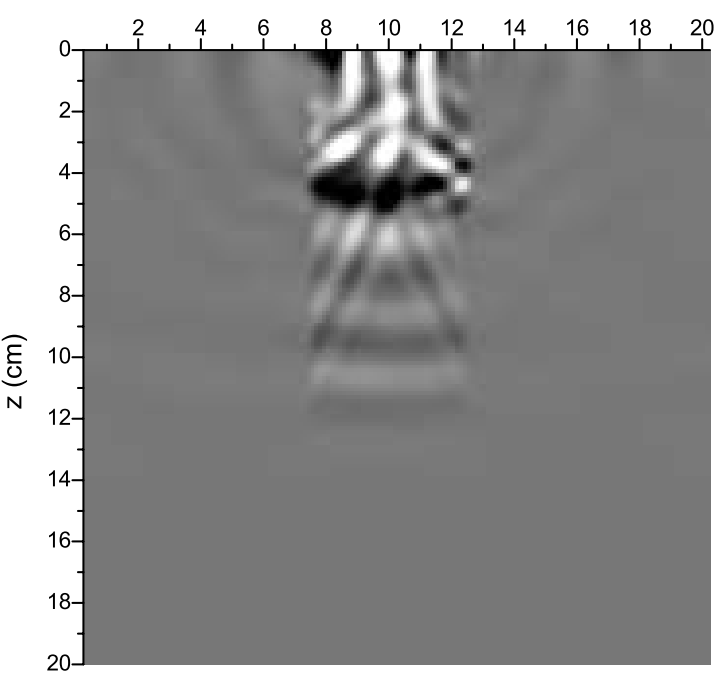

(c)

Figure 10: Model made of epoxy and zinc (a) and corresponding surface waves (b) and snapshot computed using the pseudospectral technique at a vertical section (c). 

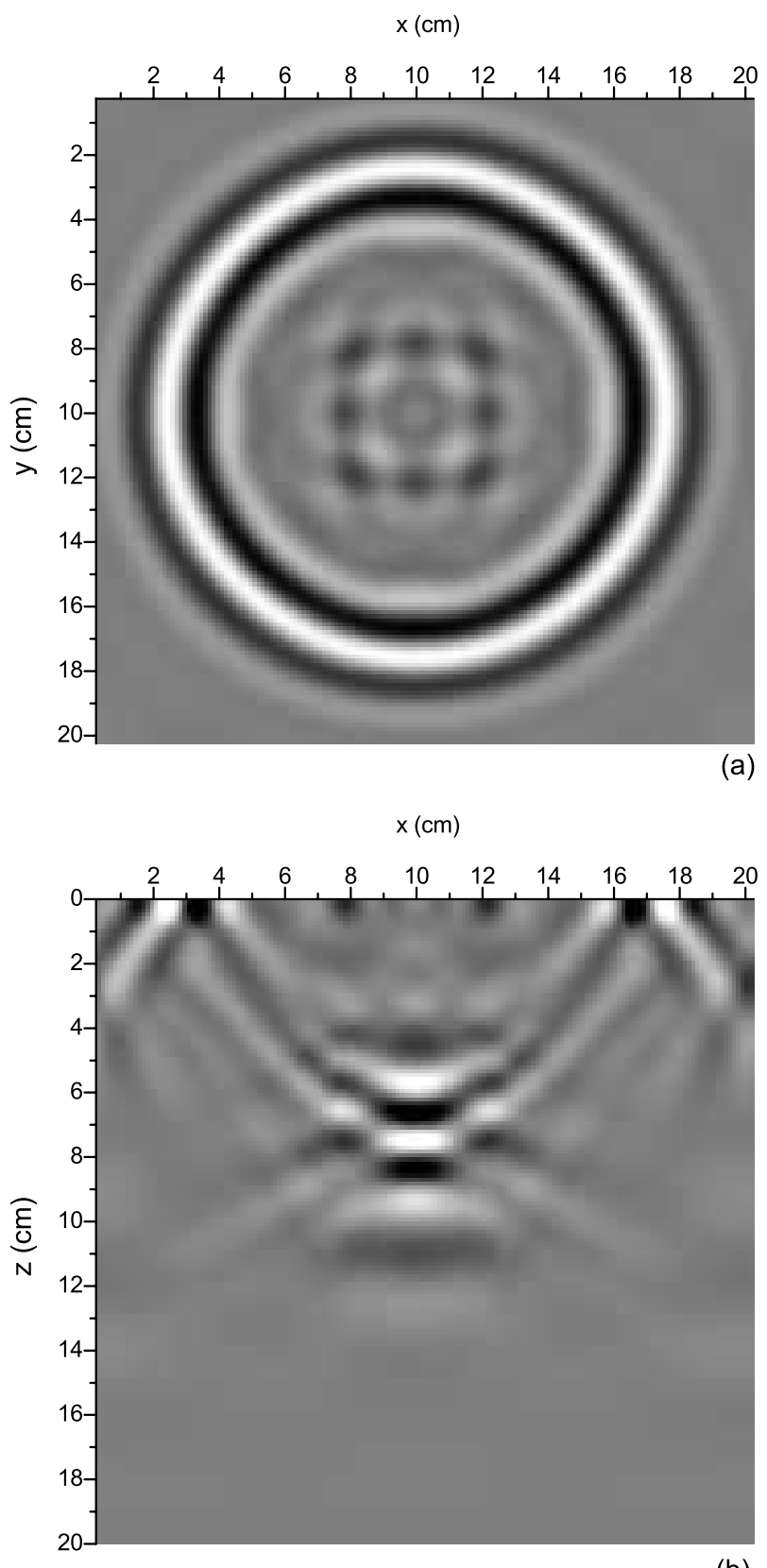

Figure 11: Surface waves (a) and snapshot computed using the pseudospectral technique at a vertical section (b) corresponding to the model shown in Figure 10a, replacing epoxy with copper. 


\section{Appendix A. Analytical solution for transversely isotropic media}

We briefly summarize the main formulas used in this article to compute the analytical vertical motion in a VTI half-space with horizontal free surface. For a thorough treatment, see Payton $[48,53]$.

\section{Appendix A.1. Step response}

Two cases are considered: $(i)$ buried source with receiver at the epicenter (idealized earthquake), and ( $i i)$ source at the surface with receiver in its vertical (idealized geophysical exploration or laboratory experiment). The two problems are mathematically related through the reciprocity principle [54].

\section{Appendix A.1.1. Buried source}

When the time dependence of the source is described by Heaviside's step function, the vertical displacement at the epicenter is given by

$$
u_{z}(t)=\frac{\rho f_{z}}{4 \pi c_{44} z_{s}} u_{0}\left(\frac{\sqrt{c_{44} / \rho}}{z_{s}} t\right)
$$

where $t$ is time, $\rho$ is density, $f_{z}$ is the amplitude of the vertical body force, $c_{44}$ is an elastic constant in Voigt notation, and $z_{s}$ is the depth of the buried source. In (A.1), function $u_{0}$ depends on the type of material; for example, in the case of apatite, we have

$$
u_{0}(\theta)= \begin{cases}0 & \text { if } 0 \leq \theta \leq \alpha^{-1 / 2} \\ F\left(\omega_{3}\right) g(\theta) & \text { if } \alpha^{-1 / 2}<\theta<1 \\ 2 F\left(\omega_{3}\right) g(\theta) & \text { if } 1 \leq \theta<T_{+} \\ 2 \operatorname{Re}\left\{F(\omega)\left[\frac{1}{2}+\mathrm{i} \frac{(2-\chi) \theta^{2}+\beta-1}{2 Q(\theta)}\right]\right\} & \text { if } T_{+} \leq \theta<\infty\end{cases}
$$

where

$$
F(\omega)=\frac{2 f V}{(f-\theta) d}
$$

with

$$
\begin{aligned}
f(\omega) & =\sqrt{\frac{\chi \omega+\alpha+1-\alpha \theta^{2}}{\alpha}} \\
V(\omega) & =\left\{(1-\kappa) \omega(\omega+1)-(\chi \omega+\alpha)(\omega+1)+\alpha \theta^{2}(\omega+1)\right. \\
& \left.-\frac{1}{\kappa}\left[(\kappa-1)(\omega+1)+\alpha \theta^{2}\right]\left(\omega+1-\alpha \theta^{2}\right)\right\} \\
d(\omega) & =2(1-\kappa) \omega(\omega+1)-(\chi \omega+\alpha)(\omega+1)-\alpha \theta f .
\end{aligned}
$$

In (A.2)-(A.4), we have defined

$$
\omega=\zeta(\theta)+\mathrm{i} \nu(\theta)
$$

where

$$
\begin{aligned}
\zeta(\theta) & =\frac{1}{2 \beta}\left[\chi \theta^{2}-(\beta+1)\right] \\
\nu(\theta) & =\frac{1}{2 \beta} \sqrt{\left(4 \alpha \beta-\chi^{2}\right)\left(\theta^{2}-T_{+}^{2}\right)\left(\theta^{2}-T_{-}^{2}\right)}
\end{aligned}
$$


with

$$
T_{ \pm}^{2}=\frac{-[\chi(\beta+1)-2 \beta(\alpha+1)] \pm \sqrt{4 \beta(\alpha+\beta-\chi)(1+\alpha \beta-\chi)}}{4 \alpha \beta-\chi^{2}} .
$$

Parameters $\alpha, \beta, \chi, \kappa$ appearing in (A.2)-(A.6) represent non-dimensional elastic constants defined by

$$
\alpha=\frac{c_{33}}{c_{44}} \quad \beta=\frac{c_{11}}{c_{44}} \quad \chi=1+\alpha \beta-\left(\frac{c_{13}}{c_{44}+1}\right)^{2} \quad \kappa=\sqrt{1+\alpha \beta-\chi}
$$

where $c_{i j}$ are, as usual, the anisotropic elastic constants in the abbreviated Voigt notation. In (A.2), $g$ and $\omega_{3}$ are defined as

$$
\begin{aligned}
g(\theta) & =\frac{1}{2}-\frac{(2-\chi) \theta^{2}+\beta-1}{2 S(\theta)} \\
\omega_{3} & =\frac{\chi \theta^{2}-(\beta+1)+S(\theta)}{2 \beta}
\end{aligned}
$$

where

$$
S(\theta)=\sqrt{\left[\chi \theta^{2}-(\beta+1)\right]^{2}-4 \beta\left(\alpha \theta^{2}-1\right)\left(\theta^{2}-1\right)} .
$$

Finally, function $Q(\theta)$ appearing in (A.2) is given by

$$
Q(\theta)=\sqrt{4 \beta\left(\alpha \theta^{2}-1\right)\left(\theta^{2}-1\right)-\left[\chi \theta^{2}-(\beta+1)\right]^{2}} .
$$

Appendix A.1.2. Point load at the surface

The epicentral-axis displacement generated at $(0,0, z)$ by a vertical point load located at $(0,0,0)$ on the horizontal free surface is given by

$$
\begin{aligned}
& u_{x}(x=0, y=0, z, t)=0 \\
& u_{y}(x=0, y=0, z, t)=0 \\
& u_{z}(x=0, y=0, z, t)=\frac{h}{4 \pi c_{44} z} u_{0}\left(\frac{t}{T}\right)
\end{aligned}
$$

where $h$ is the strength of the source, $u_{0}$ is given by (A.2), and

$$
T=\frac{z}{\sqrt{c_{44} / \rho}} \text {. }
$$

Appendix A.2. Response to an arbitrary wavelet

For convenience, we denote here with $u_{\theta}$ any one of the displacements (A.1) or (A.7), which are due to a source whose time dependence is described by the step function $\theta$. Likewise, let $u_{\phi}$ be the displacement due to an arbitrary wavelet $\phi(t)$. Since the relationship between $\phi$ and $u_{\phi}$ is linear and time invariant, there exists a function $G$ such that

$$
u_{\phi}=G * \phi
$$

where the asterisk denotes time convolution. Putting $\phi=\delta$ in (A.8) yields $G=u_{\delta}$ and hence

$$
u_{\phi}=u_{\delta} * \phi
$$

from which, in particular, we get

$$
u_{\theta}=u_{\delta} * \theta
$$


Thus, given any point source, the vertical displacement and velocity can be computed from the step-response displacement by using

$$
\begin{gathered}
u_{\phi}=u_{\theta} * \frac{\mathrm{d} \phi}{\mathrm{d} t} \\
V_{\phi}=u_{\theta} * \frac{\mathrm{d}^{2} \phi}{\mathrm{d} t^{2}} .
\end{gathered}
$$

\section{References}

[1] N. Favretto-Cristini, D. Komatitsch, J. M. Carcione, F. Cavallini, Elastic surface waves in crystals - Part 1: Review of the physics, Ultrasonics (2011) Submitted.

[2] F. R. Rollins Jr, T. C. Lim, G. W. Farnell, Ultrasonic reflectivity and surface wave phenomena on surfaces of copper single crystals, Applied Physics Letters 12 (7) (1968) 236-238.

[3] F. R. Rollins Jr, Ultrasonic examination of liquid-solid boundaries using a right-angle reflector technique, J. Acoust. Soc. Am. 44 (2) (1968) 431-434.

[4] A. A. Kolomenskii, A. A. Maznev, Phonon-focusing effect with laser-generated ultrasonic surface waves, Physical Review B 48 (19) (1993) 14502-14512.

[5] A. G. Every, K. Y. Kim, A. A. Maznev, Surface dynamic response functions of anisotropic solids, Ultrasonics 36 (1998) 349-353.

[6] A. G. Every, M. Deschamps, Principal surface wave velocities in the point focus acoustic materials signature $\mathrm{V}(\mathrm{z})$ of an anisotropic solid, Ultrasonics 41 (2003) 581-591.

[7] J. X. Dessa, G. Pascal, Combined traveltime and frequency-domain seismic waveform inversion: a case study on multi-offset ultrasonic data, Geophys. J. Int. 154 (1) (2003) 117-133. doi:10.1046/j.1365-246X.2003.01956.x.

[8] A. N. Darinskii, M. Weihnacht, Acoustic waves in bounded anisotropic media: theorems, estimations, and computations, IEEE Transactions on Ultrasonics, Ferroelectrics, and Frequency Control 52 (5) (2005) 792-801.

[9] K. Helbig, Foundations of anisotropy for exploration seismics, in: K. Helbig, S. Treitel (Eds.), Handbook of Geophysical exploration, section I: Seismic exploration, Vol. 22, Pergamon, Oxford, England, 1994.

[10] S. Crampin, E. M. Chesnokov, R. G. Hipkin, Seismic anisotropy - the state of the art II, Geophys. J. Roy. Astron. Soc. 76 (1984) 1-16.

[11] L. Thomsen, Weak elastic anisotropy, Geophysics 51 (1986) 1954-1966.

[12] J. M. Carcione, Wave fields in real media: Theory and numerical simulation of wave propagation in anisotropic, anelastic, porous and electromagnetic media, 2nd Edition, Elsevier Science, Amsterdam, The Netherlands, 2007.

[13] E. Tessmer, 3-D seismic modelling of general material anisotropy in the presence of the free surface by a Chebyshev spectral method, Geophys. J. Int. 121 (1995) 557-575.

[14] D. Komatitsch, F. Coutel, P. Mora, Tensorial formulation of the wave equation for modelling curved interfaces, Geophys. J. Int. 127 (1) (1996) 156-168. doi:10.1111/j.1365246X.1996.tb01541.x. 
[15] A. T. Patera, A spectral element method for fluid dynamics: laminar flow in a channel expansion, J. Comput. Phys. 54 (1984) 468-488.

[16] D. Komatitsch, J. P. Vilotte, The spectral-element method: an efficient tool to simulate the seismic response of 2D and 3D geological structures, Bull. Seismol. Soc. Am. 88 (2) (1998) 368-392.

[17] R. Vai, J. M. Castillo-Covarrubias, F. J. Sánchez-Sesma, D. Komatitsch, J. P. Vilotte, Elastic wave propagation in an irregularly layered medium, Soil Dynamics and Earthquake Engineering 18 (1) (1999) 11-18. doi:10.1016/S0267-7261(98)00027-X.

[18] G. Cohen, Higher-order numerical methods for transient wave equations, Springer-Verlag, Berlin, Germany, 2002.

[19] M. O. Deville, P. F. Fischer, E. H. Mund, High-Order Methods for Incompressible Fluid Flow, Cambridge University Press, Cambridge, United Kingdom, 2002.

[20] J. Tromp, D. Komatitsch, Q. Liu, Spectral-element and adjoint methods in seismology, Communications in Computational Physics 3 (1) (2008) 1-32.

[21] J. M. Carcione, D. Kosloff, R. Kosloff, Wave propagation simulation in an elastic anisotropic (transversely isotropic) solid, Q. J. Mech. Appl. Math. 41 (3) (1988) 319-345.

[22] J. M. Carcione, D. Kosloff, A. Behle, G. Seriani, A spectral scheme for wave propagation simulation in 3-D elastic-anisotropic media, Geophysics 57 (12) (1992) 1593-1607.

[23] D. Kosloff, D. Kessler, A. Q. Filho, E. Tessmer, A. Behle, R. Strahilevitz, Solution of the equations of dynamic elasticity by a Chebychev spectral method, Geophysics 55 (1990) 734-748. doi:10.1190/1.1442885.

[24] J. M. Carcione, F. Poletto, D. Gei, 3-D wave simulation in anelastic media using the Kelvin-Voigt constitutive equation, J. Comput. Phys. 196 (2004) 282-297.

[25] B. Lombard, J. Piraux, Numerical treatment of two-dimensional interfaces for acoustic and elastic waves, J. Comput. Phys. 195 (1) (2004) 90-116. doi:10.1016/j.jcp.2003.09.024.

[26] P. Moczo, J. Robertsson, L. Eisner, The finite-difference time-domain method for modeling of seismic wave propagation, in: R.-S. Wu, V. Maupin (Eds.), Advances in wave propagation in heterogeneous media, Vol. 48 of Advances in Geophysics, Elsevier - Academic Press, London, UK, 2007, Ch. 8, pp. 421-516.

[27] K. van Wijk, D. Komatitsch, J. A. Scales, J. Tromp, Analysis of strong scattering at the micro-scale, J. Acoust. Soc. Am. 115 (3) (2004) 1006-1011. doi:10.1121/1.1647480.

[28] S. J. Lee, H. W. Chen, Q. Liu, D. Komatitsch, B. S. Huang, J. Tromp, Three-dimensional simulations of seismic wave propagation in the Taipei basin with realistic topography based upon the spectral-element method, Bull. Seismol. Soc. Am. 98 (1) (2008) 253-264. doi:10.1785/0120070033.

[29] N. Favier, S. Chevrot, D. Komatitsch, Near-field influences on shear wave splitting and traveltime sensitivity kernels, Geophys. J. Int. 156 (3) (2004) 467-482. doi:10.1111/j.1365246X.2004.02178.x. 
[30] G. Seriani, E. Priolo, A. Pregarz, Modelling waves in anisotropic media by a spectral element method, in: G. Cohen (Ed.), Proceedings of the third international conference on mathematical and numerical aspects of wave propagation, SIAM, Philadephia, PA, 1995, pp. 289-298.

[31] D. Komatitsch, C. Barnes, J. Tromp, Simulation of anisotropic wave propagation based upon a spectral element method, Geophysics 65 (4) (2000) 1251-1260. doi:10.1190/1.1444816.

[32] S. Chevrot, N. Favier, D. Komatitsch, Shear wave splitting in three-dimensional anisotropic media, Geophys. J. Int. 159 (2) (2004) 711-720. doi:10.1111/j.1365-246X.2004.02432.x.

[33] D. Komatitsch, L. P. Vinnik, S. Chevrot, SHdiff/SVdiff splitting in an isotropic Earth, J. Geophys. Res. 115 (B7) (2010) B07312. doi:10.1029/2009JB006795.

[34] D. Komatitsch, D. Michéa, G. Erlebacher, Porting a high-order finite-element earthquake modeling application to NVIDIA graphics cards using CUDA, Journal of Parallel and Distributed Computing 69 (5) (2009) 451-460. doi:10.1016/j.jpdc.2009.01.006.

[35] D. Komatitsch, G. Erlebacher, D. Göddeke, D. Michéa, High-order finite-element seismic wave propagation modeling with MPI on a large GPU cluster, J. Comput. Phys. 229 (20) (2010) 7692-7714. doi:10.1016/j.jcp.2010.06.024.

[36] D. Komatitsch, Fluid-solid coupling on a cluster of GPU graphics cards for seismic wave propagation, Comptes Rendus de l'Académie des Sciences - MécaniqueIn press.

[37] P. Micikevicius, 3D finite-difference computation on GPUs using CUDA, in: GPGPU-2: Proceedings of the 2nd Workshop on General Purpose Processing on Graphics Processing Units, Washington, DC, USA, 2009, pp. 79-84. doi:10.1145/1513895.1513905.

[38] D. Michéa, D. Komatitsch, Accelerating a 3D finite-difference wave propagation code using GPU graphics cards, Geophys. J. Int. 182 (1) (2010) 389-402. doi:10.1111/j.1365246X.2010.04616.x.

[39] D. Komatitsch, R. Martin, An unsplit convolutional Perfectly Matched Layer improved at grazing incidence for the seismic wave equation, Geophysics 72 (5) (2007) SM155-SM167. doi:10.1190/1.2757586.

[40] R. Martin, D. Komatitsch, S. D. Gedney, A variational formulation of a stabilized unsplit convolutional perfectly matched layer for the isotropic or anisotropic seismic wave equation, Comput. Model. Eng. Sci. 37 (3) (2008) 274-304.

[41] R. Martin, D. Komatitsch, A. Ezziani, An unsplit convolutional perfectly matched layer improved at grazing incidence for seismic wave equation in poroelastic media, Geophysics 73 (4) (2008) T51-T61. doi:10.1190/1.2939484.

[42] C. Canuto, M. Y. Hussaini, A. Quarteroni, T. A. Zang, Spectral methods in fluid dynamics, Springer-Verlag, New-York, USA, 1988.

[43] E. Priolo, J. M. Carcione, G. Seriani, Numerical simulation of interface waves by high-order spectral modeling techniques, J. Acoust. Soc. Am. 95 (2) (1994) 681-693.

[44] T. J. R. Hughes, The finite element method, linear static and dynamic finite element analysis, Prentice-Hall International, Englewood Cliffs, New Jersey, USA, 1987. 
[45] N. Tarnow, J. C. Simo, How to render second-order accurate time-stepping algorithms fourth-order accurate while retaining the stability and conservation properties, Comput. Meth. Appl. Mech. Eng. 115 (1994) 233-252.

[46] T. Nissen-Meyer, A. Fournier, F. A. Dahlen, A 2-D spectral-element method for computing spherical-earth seismograms - II. Waves in solid-fluid media, Geophys. J. Int. 174 (2008) 873-888. doi:10.1111/j.1365-246X.2008.03813.x.

[47] A. Maznev, A. M. Lomonosov, P. Hess, A. A. Kolomenskii, Anisotropic effects in surface acoustic wave propagation from a point source in a crystal, The European Physical Journal B 35 (2003) 429-439.

[48] R. G. Payton, Elastic wave propagation in transversely isotropic media, Martinus Nijhoff, The Hague, The Netherlands, 1983.

[49] O. Poncelet, M. Deschamps, A. Every, B. Audoin, Extension to cuspidal edges of wave surfaces of anisotropic solids: treatment of near cusp behavior, Review of Progress in Quantitative Nondestructive Evaluation 20 (2001) 51-58.

[50] M. Deschamps, O. Poncelet, Inhomogeneous plane wave and the most energetic complex ray, Ultrasonics 40 (2002) 293-296.

[51] M. Deschamps, G. Huet, Complex surface rays associated with inhomogeneous skimming and Rayleigh waves, Int. J. Nonlinear Mech. 44 (2009) 469-477.

[52] J. M. Carcione, Staggered mesh for the anisotropic and viscoelastic wave equation, Geophysics 64 (1999) 1863-1866.

[53] R. G. Payton, Epicenter and epicentral-axis motion of a transversely isotropic elastic halfspace, SIAM J. Appl. Math. 40 (1981) 373-389.

[54] A. Ben-Menahem, S. J. Singh, Seismic waves and sources, Springer-Verlag, New York, 1981. 